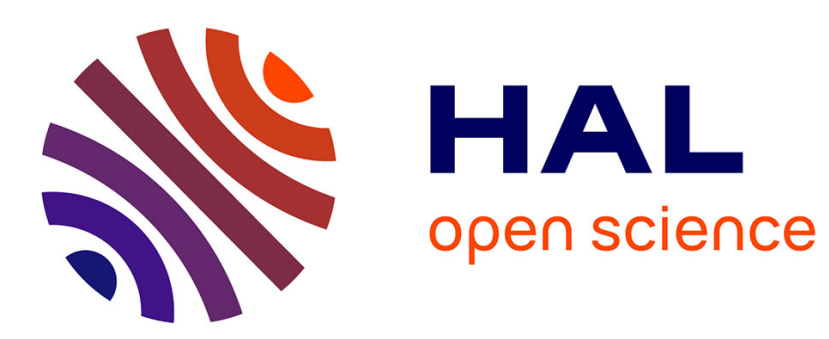

\title{
Using Good Vibrations: Melting and Controlled Shear Jamming of Dense Granular Suspensions
}

C Garat, Sébastien Kiesgen de Richter, P Lidon, A Colin, G. Ovarlez

\section{To cite this version:}

C Garat, Sébastien Kiesgen de Richter, P Lidon, A Colin, G. Ovarlez. Using Good Vibrations: Melting and Controlled Shear Jamming of Dense Granular Suspensions. 2021. hal-03356285

\section{HAL Id: hal-03356285 \\ https://hal.science/hal-03356285}

Preprint submitted on 27 Sep 2021

HAL is a multi-disciplinary open access archive for the deposit and dissemination of scientific research documents, whether they are published or not. The documents may come from teaching and research institutions in France or abroad, or from public or private research centers.
L'archive ouverte pluridisciplinaire HAL, est destinée au dépôt et à la diffusion de documents scientifiques de niveau recherche, publiés ou non, émanant des établissements d'enseignement et de recherche français ou étrangers, des laboratoires publics ou privés. 


\section{Using Good Vibrations:}

\section{Melting and Controlled Shear Jamming of Dense Granular Suspensions}

C. Garat, ${ }^{1,}$ a) S. Kiesgen de Richter, ${ }^{2}$ P. Lidon, ${ }^{1}$ A. Colin, ${ }^{3}$ and G. Ovarlez ${ }^{1}$

${ }^{1)}$ Univ. Bordeaux, CNRS, Solvay, LOF, UMR 5258, F-33608 Pessac, France

${ }^{2)}$ Université de Lorraine, CNRS, LEMTA, F-54000 Nancy, France

${ }^{3)}$ ESPCI Paris, PSL Research University, MIE-CBI, CNRS UMR 8231, 10, Rue Vauquelin, F-75231 Paris Cedex 05, France

(Dated: September 27, 2021)

Flows of suspensions can be blocked when the suspended particles are densely packed. This makes their formulation and their transport challenging in the industry. In this paper, we study the impact of vibrations on the behavior of dense granular suspensions prepared at volume fraction above their jamming volume fraction, but below the particle assembly random close packing. Vibrations are shown to have a strong effect on their rheological properties and to tune their transition from solid-like to liquid-like behavior. We study suspensions of rough silica particles in a Newtonian fluid. In the absence of vibrations, they have a solid-like behavior: they flow only above a yield stress. Particles are confined by the liquid interface and the yield stress is of frictional origin. When vibrations are applied, the yield stress vanishes to give rise to a liquid-like pseudo-Newtonian behavior at low shear rate. Using shear-reversal experiments, we show that these liquid-like vibrated suspensions of frictional particles behave like nonvibrated suspensions of frictionless particles. As the shear rate is increased, we observe a shear thickening of the vibrated suspensions, eventually leading to shearjamming: the yield stress behavior is recovered and vibrations have no more impact. We show that this shear thickening can be tuned by changing the vibration energy injected into the system. We finally propose a physical picture based on the competition between contact opening by vibration and contact formation by shear to account for these behaviors. In the framework of the Wyart and Cates (2014) model, vibrations can be seen as introducing a thermal-like repulsive force, yielding a critical stress proportional to the vibration stress introduced by Hanotin et al. (2015).

\section{INTRODUCTION}

\section{A. Dense suspensions and jamming}

Granular suspensions are suspensions of non-Brownian particles immersed in a Newtonian fluid at a high volume fraction, close to their jamming transition. The main challenge with these suspensions in the industry, e.g., when dealing with civil engineering materials such as cement pastes [1] or foodstuff such as chocolate pastes [2], is to be able to handle suspensions that are as concentrated as possible.

In these materials, the particle size is large enough ( $>10 \mu \mathrm{m}$ ) for thermal fluctuations to be neglected, and hydrodynamic interactions are in competition with contact forces. Despite their apparent simplicity, they exhibit diverse nonNewtonian behaviors, including yielding, shear thickening, normal stress differences and shear jamming [3, 4]. Mainly volume-imposed rheology is used to characterize their behavior, in which case the control parameter is the particle volume fraction $\phi$. In this framework, the rheology is described by the shear stress $\sigma$ and the normal stress differences, which both depend on $\phi$ and on the shear rate $\dot{\gamma}$. The basic manifestation of jamming in these systems is that their apparent viscosity $\eta(\dot{\gamma}, \phi)=\sigma / \dot{\gamma}$ increases strongly with $\phi$ and diverges close to the jamming point $J$, characterized by a volume fraction $\phi_{j}$. Attempts to formulate materials beyond $\phi_{j}$ then leads to the formation of solid-like granules, a process known as wet granulation in the industry [5].

\footnotetext{
a)Electronic mail: chloe.garat-ext@ solvay.com
}

An alternative view on $\phi_{j}$ comes from pressure-imposed rheology $[6,7]$, for which the control parameter is the particle normal stress $p$ (often gravity-induced). In such case, the material volume fraction $\phi$ is free to adjust to the flow conditions and its flow resistance is characterized by its friction coefficient $\mu=\sigma / p$. For a given applied $p, \mu$ increases with the applied shear rate $\dot{\gamma}$, whereas $\phi$ decreases with $\dot{\gamma}$. The densest possible flowing state, characterized by $\phi_{j}$, is thus reached here in the limit of quasistatic flows. This is reminiscent of the critical state of dry granular materials [8], characterized by a volume fraction $\phi_{c}$ and an internal friction coefficient $\mu_{c}$ in the quasistatic limit. For dry granular media and granular suspensions made of the same particles, the critical/jamming volume fractions $\phi_{c}$ and $\phi_{j}$ are equal [7]; they also have the same quasistatic friction coefficient. This suggests that interparticle friction plays the main role in both materials.

A dense granular material $\left(\phi>\phi_{c}\right)$, once sheared under a constant normal stress, is known dilate to reach the critical value $\phi_{c}$ at steady state [9]. By contrast, a loose system $\left(\phi<\phi_{c}\right)$ contracts to reach $\phi_{c}$ under shear. Coming back to the volume-imposed rheology of dense suspensions, for $\phi>\phi_{j}$, the only way for a suspension to flow would then be to dilate; however, as the volume is imposed, the system is confined and jamming occurs. In practice, this confinement can be due to the liquid interface surface tension, as shown for granulation [5].

For industrial applications, the value of $\phi_{j}$ should finally be as high as possible: its possible control through changes in the mix-design or in the process requires an in-depth understanding of the physics involved. As reviewed below, interparticle contacts play the major role in the suspension macroscopic properties close to $J$. 


\section{B. Jamming and interparticle friction}

The hydrodynamic and contact contributions to the rheology of suspensions have been studied by Gallier et al. [10] and Chèvremont, Chareyre, and Bodiguel [11]. At low concentration, hydrodynamic interactions are dominant. As the concentration increases, the contribution of contact forces is observed to increase and to overcome that of hydrodynamic forces. Close to the jamming transition, contact interactions provide the dominant contribution to the suspension stresses and govern their behavior [3], in particular the value of $\phi_{j}$.

In general for monodisperse spheres, the jamming fraction $\phi_{j}$ is strongly dependent on the shape, polydispersity and sliding friction coefficient $\mu_{p}$ of particles [4]. For monodisperse spheres, $\phi_{j}\left(\mu_{p}\right)$ is a monotonic decreasing function of $\mu_{p}$ [12]. The value of $\phi_{j}$ can then range from the random close packing (RCP) $\phi_{j}^{\mu_{p}=0}=\phi_{\text {rcp }}=0.64$ for frictionless spheres to the random loose packing (RLP) $\phi_{j}^{\mu_{p} \rightarrow \infty}=\phi_{\mathrm{rlp}}=0.55$ for highly frictional spheres. It is consistent with simulations of dry frictionless spheres flows showing that the value of the critical volume fraction $\phi_{c}$ is equal to $\phi_{\text {rcp }}$ [13], and with experiments using bubbles as an analogue of a suspension of frictionless particles, which found a value of $\phi_{j}$ close to $\phi_{\text {rcp }}$ [14].

The dependence of $\phi_{j}$ with interparticle friction coefficient plays a major role in the shear thickening - that is, an increase of $\eta$ with $\dot{\gamma}$-often reported close to $J$ in these systems [1519]. It has been proposed to relate this viscosity increase to an increase of the number of interparticle contacts upon shear in the suspension. Such behavior can be attributed, e.g., to the existence of a repulsive force between the particles [17, 19]: in that case, there are no direct contacts at low shear stress, as the particles cannot approach each others, whereas the hydrodynamic normal forces become sufficiently important at high load to bring particles into frictional contact. In the framework discussed above, an increase of the fraction of frictional contacts with the applied stress $\sigma$ implies a decrease of $\phi_{j}$ with $\sigma$. For a suspension of fixed volume fraction $\phi$ smaller that $\phi_{j}$ at rest, $\phi$ then gets closer to the stress-dependent jamming point $\phi_{j}(\sigma)$ as $\sigma$ is increased, and the material viscosity increases, i.e., the suspension shear thickens. In some cases, above a critical stress $\sigma_{c}, \phi_{j}(\sigma)$ can even be lower than $\phi$, meaning that the material cannot flow anymore: a jamming transition, called shear jamming, is then observed. We will come back in more detail to this point in Sec. IV.

\section{Tuning interparticle contacts}

In industrial applications, for given particle shape and polydispersity, increasing $\phi_{j}$ requires the ability to lower the value of the interparticle friction coefficient. This is usually done by using polymer additives in the suspension $[1,20]$. Once adsorbed on the particle surface, the polymer layer creates a repulsive barrier of low friction coefficient, ensuring a high value of $\phi_{j}$ for the suspension at low stress. Shear thickening is then observed at a critical stress dependent on the magnitude of the created repulsive force barrier [19]. It is worth noting that for adhesive particles, the same polymer layer prevents the particles from agglomerating at rest [1].

For a given suspension mix design, another way to unjam the material consists in playing with the flow configuration. Indeed, as contacts exist only in compression, these contacts can be easily open by small perturbations [21]. A way to impose such perturbations consists in superimposing a small shear oscillation orthogonal to the primary shear flow, which has been shown to be able to shift the shear-thickening transition of a suspension to higher stresses [22-24]. In these last cases, macroscopic flow perturbations are considered.

Another possibility, for non-buoyant suspensions, consists in vibrating the suspension. Vibrations can indeed fluidize a jammed suspension, turning a yield stress suspension into a viscous suspension $[25,26]$. This has been shown by applying well-controlled mechanical vibrations to fluid-saturated granular suspensions made of settled glass beads [25]. At rest, the particle pressure is set by gravity and these materials have a frictional yield stress. When vibrations are applied, their yield stress is suppressed and vibrations control their viscosity: they then have a shear-thinning behavior with a Newtonian plateau at low shear stress, the viscosity of which decreases with the vibration intensity [25, 27]. This phenomenon finds its origin in the differential acceleration between the particles and the fluid: contacts are constantly renewed by vibrations. Gaudel et al. [28] have shown that the vibrations lead to a diffusive motion of the particles, which explains the observation of a Newtonian behaviour. The average number of contacts, and thus the viscosity of the sheared suspension, is then assumed to result from a subtle interplay between contact formation by shear and contact breaking by vibration [26].

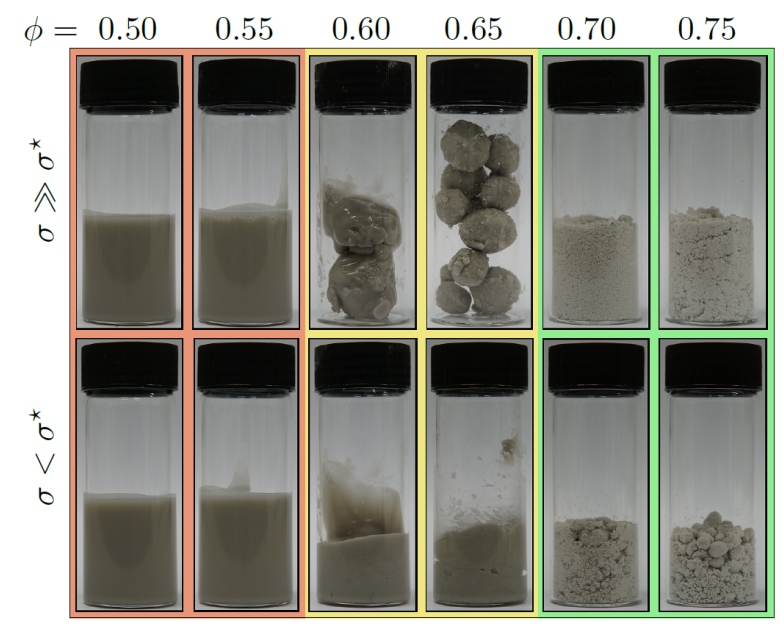

FIG. 1. Figure 1 from Hodgson et al. [5] showing the result of (upper line) strongly mixing suspensions of repulsive glass spheres in a mixture of glycerol and water at different volume fractions $\phi$ and (bottom line) subsequently vibrating the suspensions with a low stress vortex mixer. The first two columns correspond to $\phi<\phi_{m}$. The next two columns correspond to $\phi_{m}<\phi<\phi_{\text {rcp. }}$. The last two columns correspond to $\phi>\phi_{\text {rcp. }}$. The studied suspension is characterized by $\phi_{m}=0.57$ and $\phi_{\text {rcp }}=0.66$. 
This impact of vibrations also appears when preparing suspensions and studying granulation. E.g., Hodgson et al. [5] report the result of mixing a suspension of particles at different volume fractions with a high-shear mixer and of subsequently vibrating the suspension with a low stress vortex mixer (see Fig. 1). Defining $\phi_{m}$ as the jamming volume fraction of the frictional suspension, that is, the volume fraction at which the viscosity diverges at high stress, they observed liquid-like suspensions in both cases when preparing the suspension for $\phi<\phi_{m}$. For $\phi_{m}<\phi<\phi_{\text {rcp }}$ the high-shear mixing produced solid-like granules, which melted to a liquidlike suspension upon vortex mixing: this is reminiscent of the vibration-induced fluidization observed by Hanotin et al. [25]. Finally, for $\phi>\phi_{\text {rcp }}$ solid-like granules from the high-shear mixing remained solid upon vortex mixing.

The suspension studied by Hodgson et al. [5] is made of repulsive particles: once particles are pulled apart with the help of vibrations, they remain separated and the system remains frictionless and liquid-like as long as shear is kept low; its low shear viscosity then diverges at $\phi_{\mathrm{rcp}}$. This is not the case when repulsive forces are negligible, as in the work of Hanotin et al. [25]: the initially jammed suspension remains fluid only as long as vibrations are kept on; turning vibrations off causes the suspension to return to a solid-like jammed state.

In the framework discussed in the present introduction, vibrations finally seem to introduce an effective thermal-like repulsive force between the particles [25, 28], which would prevent the persistence of solid contacts as long as vibrations are efficient, and would make the particles frictionless-like. This picture has to be examined closely.

\section{Outline}

In this paper, we investigate the behavior of concentrated suspensions of non-repulsive frictional particles prepared in their jammed state, i.e., at $\phi_{m}<\phi<\phi_{\text {rcp }}$, where $\phi_{m}$ is the jamming volume fraction for the frictional particles in the absence of vibrations, with the aim of understanding how vibrations affect their behavior under flow. Our purpose is to see to what extent the material can be unjammed, and to observe in which conditions a re-entrant (shear-)jamming transition can be observed. We also want to explore carefully the analogy between vibrated and frictionless particles. By tuning the amplitude and frequency of the vibrations, i.e., the effective repulsive force between the particles, we finally aim at providing data for testing models of nonlinear rheology of suspensions.

We use a non-buoyant jammed suspension, where the material is simply confined by the liquid interface surface tension at rest, and keeps a constant volume fraction throughout the experiments under vibration. This is to be contrasted with the study of Hanotin et al. [25], who worked with a settled suspension, free to dilate: the volume fraction then resulted from a complex interplay between gravity, vibrations, and shear, but was free to vary, likely preventing shear jamming to occur. Here, we aim at working in the framework of volume-fractionimposed rheology. Moreover, we use rough irregular-shaped particles, which makes it possible to access a wide range of volume fractions in the jammed state [29].

In Sec. II we present the suspension and set-up used in this paper; we characterize the particle assembly random close packing and the jamming volume fraction of the frictional particles. In Sec. III, we show the differences between vibrated and non-vibrated suspensions, and study the impact of a change of the vibration characteristics and of the particle fraction on the behavior; we also compare the viscosity of vibrated suspensions to that of frictionless suspensions. In Sec. IV, we finally discuss the origin of the observed behavior and propose a physical picture based on those of Hanotin et al. [26] for the impact of vibrations and of Wyart and Cates [16] for the transition from frictionless to frictional particles.

We show that, in the absence of vibrations, the prepared suspensions flow only above a yield stress, which is of frictional origin. By contrast, when vibrations are applied, the yield stress vanishes to give rise to a liquid-like pseudoNewtonian behavior at low shear rate. Using shear-reversal experiments, we show that these liquid-like vibrated suspensions of frictional particles behave like nonvibrated suspensions of frictionless particles. As the shear rate is increased, we observe a shear thickening of the vibrated suspensions, eventually leading to shear-jamming; this transition is tuned by the vibration energy injected into the system, suggesting that vibrations introduce a thermal-like repulsive force.

\section{MATERIALS AND METHODS}

\section{A. Suspensions}

We study the rheological behavior of a dense granular suspension composed of polydisperse irregular-shaped silica particles (Fig. 2) immersed in a lighter Newtonian fluid. The particles (from Solvay) are made of agglomerated elementary amorphous silica particles of $18 \mathrm{~nm}$ diameter. These particles are first organized into a dense fractal network of fractal dimension $D_{f_{1}}=2.6$ at a size of $50 \mathrm{~nm}$; these primary aggregates are then agglomerated at a larger scale to form the particles [30]. Cryo-SEM images of the surface of single particles (Fig. 2) suggest that the particle roughness is tuned by the size of the primary aggregates. The particle volumeweighted mean diameter, measured by laser granulometry is $30 \mu \mathrm{m}$ (Fig. 2d); the width of the volume distribution can be estimated as $\left(d_{90}-d_{10}\right) / d_{50}=2.7$, where $d_{x}$ denotes the particle size below which $x \%$ of the sample is contained.

The density of the elementary silica particles is 2.1 . The effective density of the agglomerated particles is much lower. When immersed, they trap some fluid and their density is of order 1.3, as estimated below.

The fluid is a water solution of $\mathrm{Na}_{2} \mathrm{SO}_{4}$ at a $0.5 \mathrm{wt} \%$ concentration. This salt comes from the dissolution of $\mathrm{Na}_{2} \mathrm{SO}_{4}$ that was precipitated on the particles during the industrial fabrication process. The fluid viscosity is $9.5 \cdot 10^{-4} \mathrm{~Pa} . \mathrm{s}$, the fluid density is 1 , and the surface tension is $\Gamma_{t}=0.07 \mathrm{~N} . \mathrm{m}^{-1}$, at a temperature of $25^{\circ} \mathrm{C}$ at which the experiments are performed. 

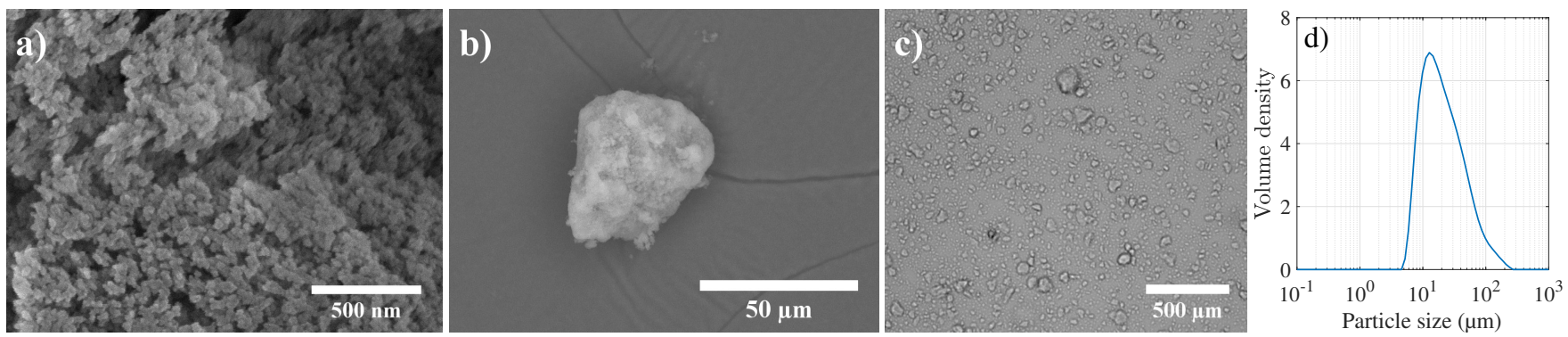

FIG. 2. a) Cryo-SEM image of the surface of a suspended silica particle. b) SEM image of a dry silica particle. c) Optical microscopy of a dilute silica suspension. The scale bars are on the pictures. The elementary particles and the primary aggregates are observable in Fig. 2a. d) Volume-weighted size distribution of the silica particles measured by laser granulometry; in addition, $6 \%$ of the particles in the suspension, not seen here, are primary aggregates of $90 \mathrm{~nm}$ mean diameter (see main text).

Dense suspensions are prepared by mixing silica with demineralized water at various initial mass fractions $\phi^{\mathrm{wt}} \geq$ $24 \mathrm{wt} \%$, for which the material has a yield stress at rest (i.e., it is jammed). Preparing homogeneous materials of concentration above $24 \mathrm{wt} \%$ is impossible by mechanical mixing, as air is trapped into the jammed material through dilation and fractures, leading to the formation of granules (see Fig. 3). As discussed in Sec. IC, this $24 \mathrm{wt} \%$ fraction is thus close to the limit of granulation, which is also the jamming fraction of frictional suspensions $\phi_{m}^{\mathrm{wt}}$ as shown by Hodgson et al. [5].

Concentrations above $24 \mathrm{wt} \%$ are achieved by mixing with a vortex applying vibrations (Fig. 3). This is a first indication that the material, initially jammed, can be unjammed by vibrations. Note that the particles are slightly eroded by the vortex mixing, leading to the presence of primary aggregates in the suspension. To ensure that the same particle assembly is characterized in all cases, we limit our study to suspensions that were all vortex-mixed for the same duration $-150 \mathrm{~s}-$ at the same (maximum) rate, which corresponds to a vibration frequency of $45 \mathrm{~Hz}$ with an amplitude of order $5 \mathrm{~mm}$. This corresponds to a vibration energy (see below) 100 times higher than that injected in the suspension in the rheological experiments. In these conditions, the primary aggregates fraction was estimated to be $6 \%$ of the total particle volume by dry mass measurement of the supernatant formed after centrifugation of the suspension; their mean diameter is $90 \mathrm{~nm}$, as measured by laser granulometry on the supernatant. In these experimental conditions, it was not possible to prepare liquidlike materials at fractions beyond $29 \mathrm{wt} \%$ (Fig. 3), which is thus likely close to RCP for our suspensions, by analogy with the observations of Hodgson et al. [5].

In order to account for the particle wear by vortex mixing and to study the rheology of suspensions of particle all made of the same particles, all suspension samples were first prepared at the maximum possible mass fraction by dispersing $29 \mathrm{wt} \%$ of particles in water, and by vibrating the suspension during $150 \mathrm{~s}$ with the vortex mixer. The sample was then diluted to achieve the mass fraction $\phi^{\mathrm{wt}}$ for which the rheology is studied. We checked that subsequent low energy vibration and flow with the rheometer coupled to the vibration exciter (see below) did not cause any additional particle wear and did not affect further the particle size distribution. This

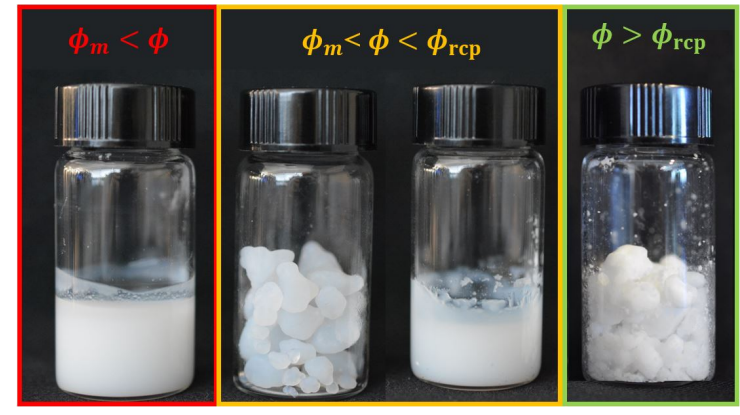

FIG. 3. Result of mixing suspensions of silica particles at different mass fractions $\phi^{\mathrm{wt}}$. From left to right, the first vial corresponds to $\phi^{\mathrm{wt}}<\phi_{m}^{\mathrm{wt}}$ with and without vibrations (here, $\phi^{\mathrm{wt}}=23 \mathrm{wt} \%$ and vibrations are not applied). The second and third vials correspond to $\phi_{m}^{\mathrm{wt}}<\phi^{\mathrm{wt}}<\phi_{r c p}^{\mathrm{wt}}$ (here, $\phi^{\mathrm{wt}}=29 \mathrm{wt} \%$ ), without vibrations (second vial) or when vibrations are continuously applied during mixing (third vial). The last vial corresponds to $\phi^{\mathrm{wt}}>\phi_{\text {rcp }}^{\mathrm{wt}}$ with and without vibrations (here, $\phi^{\mathrm{wt}}=30 \mathrm{wt} \%$ and vibrations are applied). The studied suspension is characterized by $\phi_{m}^{\mathrm{wt}} \simeq 0.24$ and $\phi_{\text {rcp }}^{\mathrm{wt}} \simeq 0.3$.

was checked by comparing the particle distribution in three $29 \mathrm{wt} \%$ samples: one was sheared at $10 \mathrm{~s}^{-1}$ for $10 \mathrm{~min}$, one was vibrated for $10 \mathrm{~min}$ at the highest vibration energy achievable with the vibration exciter, while the last sample was kept at rest. The same amount of primary aggregates was found in all samples, confirming that vibrations and flow did not affect the particles size distribution. Consistently, we have observed that the impact of vibrations on the behavior is reversible.

We have characterized the behavior of the liquid-like suspensions for various $\phi^{\mathrm{wt}} \leq 24 \mathrm{wt} \%$ (see appendix A, Figs. 14 and 15): their viscosity measured at a given shear stress $(0.01 \mathrm{~Pa})$ tends to diverge at $\phi^{\mathrm{wt}} \simeq 0.25$. This value is consistent with the limit of granulation discussed above. In addition, the flow curves of Fig. 14 suggest that, by contrast with the suspension of Hodgson et al. [5], repulsion between the particles is negligible (otherwise, shear thickening would have been observed in the densest suspensions, with a low viscosity plateau diverging at $\phi_{\mathrm{rcp}}^{\mathrm{wt}}$ ): we deal here with a frictional suspension at any observable shear stress.

Finally, from all the above observations, we estimate the values of the jamming fraction of the frictional and friction- 
less suspensions to be, respectively, $\phi_{m}^{\mathrm{wt}} \simeq 0.24$ and $\phi_{\mathrm{rcp}}^{\mathrm{wt}} \simeq 0.3$. All of our experiments were performed for $\phi_{m}^{\mathrm{wt}}<\phi^{\mathrm{wt}}<\phi_{\mathrm{rcp}}^{\mathrm{wt}}$, that is, in the range of mass fraction where the frictional suspension is a priori jammed.

Note that there is a wide range of accessible mass fractions for the frictional jammed states, from $24 \mathrm{wt} \%$ to $30 \mathrm{wt} \%$. This is a key point for investigating the transition from frictionless to frictional states leading to shear-jamming, as preparing a jammed state is easier and less sensitive to slight changes in the composition. Computing the ratio $\phi_{j}\left(\mu_{p}\right) / \phi_{j}\left(\mu_{p}=0\right)=$ $\phi_{m} / \phi_{\mathrm{rcp}}=\phi_{m}^{\mathrm{wt}} / \phi_{\mathrm{rcp}}^{\mathrm{wt}}$, we get here $\phi_{m} / \phi_{\mathrm{rcp}} \simeq 0.8$, whereas $\phi_{m} / \phi_{\text {rcp }} \gtrsim 0.9$ for frictional spheres. This likely comes from the irregular shape and the large effective roughness of the studied particles. Indeed, $\phi_{m} / \phi_{\text {rcp }} \simeq 0.8$ is also found for cornstarch particles [31]. This is consistent with numerical simulations of dry granular materials and suspensions which show that (i) frictional polygons with a small number of edges behave like frictional discs of high rolling resistance [32], and that (ii) the critical state volume fraction of frictional discs and the jamming volume fraction of frictional spheres are decreased when adding rolling resistance [29, 33].

Assuming that the RCP mass fraction $\phi_{\text {rcp }}^{\mathrm{wt}} \simeq 0.3$ corresponds to a volume fraction of order $70 \%$ for the studied polydisperse particles, we finally estimate that, when suspended in water, they are composed of $25 \%$ of agglomerated silica particles and of $75 \%$ of entrapped fluid. This leads to an effective density of the particles of order 1.3.

\section{B. Rheometry}

\section{Rheometry under vibrations}

Rheological measurements have been obtained using the same original set-up (Fig. 4) as Gaudel et al. [28], which couples a stress-imposed rheometer (AR1500ex, TA instruments) with a vibration exciter (Brüel \& Kjær); additional experiments on non-vibrated suspensions have been performed with a Kinexus Ultra+ rheometer (Netzsch). The suspension is first poured in a cup of $50 \mathrm{~mm}$ diameter equipped with $4 \mathrm{~mm}$ baffles to avoid wall slip. A six blades vane $(22 \mathrm{~mm}$ diameter, $50 \mathrm{~mm}$ length) is then inserted into the material and rotated to characterize its behavior. The use of a vane tool instead of a Couette bob allows the tool insertion in the jammed material and prevents from wall slip. The cup is connected to a vibration shaker controlled by a power amplifier, an accelerometer and a generator. The amplitude $A$ and frequency $f$ of the applied vertical vibrations vary from $100 \mu \mathrm{m}$ to $500 \mu \mathrm{m}$ and $10 \mathrm{~Hz}$ to $50 \mathrm{~Hz}$ respectively. The relevant control parameter in vibrated granular materials and granular suspensions has been shown to be the mechanical vibration stress

$$
\sigma_{v}=\frac{1}{2} \rho_{s} A^{2}(2 \pi f)^{2}
$$

with $\rho_{s}$ the suspension density. $\sigma_{v}$ is the density of kinetic energy injected into the system [25, 26, 34].

In the experiments, the vane is not fully immersed in the suspension to avoid possible Janssen effects which would af-

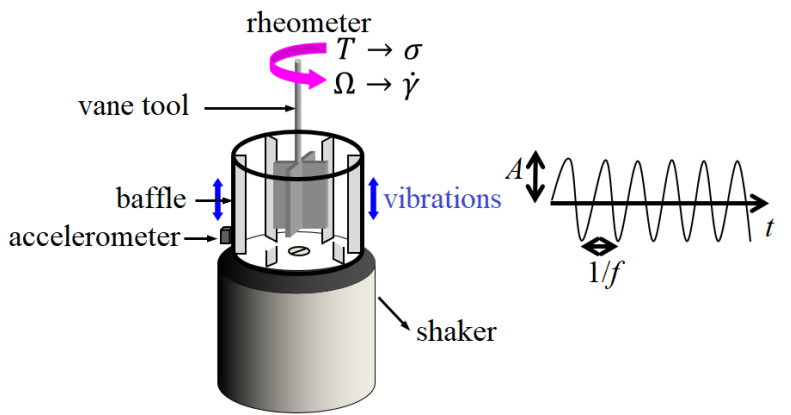

FIG. 4. Sketch of the set-up.

fect the particle pressure variation with height for frictional flows [35]; such effects are typically observed for depths larger than 1.5 times the gap size [35] when the particle pressure is set by gravity. Here the immersed length $h_{v}$ of the vane is $21 \mathrm{~mm}$ (it is shown in the appendix B that there is no observable Janssen effect at this depth). A procedure based on a Couette analogy [36] is used to extract the shear stress and shear rate from the torque and angular velocity respectively. Accordingly, the shear stress $\sigma$ is estimated by using

$$
\sigma=\frac{T}{2 \pi R^{2} h_{v}}
$$

where $T$ is the torque, and $R$ is the radius of the vane, and the shear rate is estimated as

$$
\dot{\gamma}=\frac{\Omega R}{e}
$$

where $\Omega$ is the vane tool rotation velocity, and $e=4.63 \mathrm{~mm}$ is the effective sheared gap allowing for quantitative measurements in this geometry with the Couette analogy. As discussed in the appendix B, Eq. (2) is relevant here even for frictional flows as the particle stress does not depend on depth in our system.

For the densest suspensions (28 and $29 \mathrm{wt} \%$ ), the suspension is pre-vibrated at $f=50 \mathrm{~Hz}$ and $A=500 \mu \mathrm{m}$ for $10 \mathrm{~min}$ before each measurement to ensure an initial reproducible homogeneous state, which is crucial when the material in its jammed state has been fractured by shear. The fractures are observed to disappear under vibrations, and the material surface looks homogeneous, suggesting homogeneity of the material; this is confirmed by the good reproducibility of the experiments. At lower mass fraction, to prevent from possible sedimentation, suspensions are stirred manually before each measurement.

Tests were carried out at room temperature $T \simeq 25^{\circ} \mathrm{C}$. The response of the material subjected to a logarithmic shear-rate ramp from $\dot{\gamma}=0.01$ to $100 \mathrm{~s}^{-1}$ was studied, with a ramp speed of $1 \mathrm{decade} / \mathrm{min}$, for various amplitudes and frequency of vibrations. As shown below, the material behavior in its jammed state is characterized by a plastic plateau, typical of granular frictional flows, which cannot be captured accurately under controlled stress [37]: we thus chose to control the shear rate. 


\section{Shear-reversal experiments}

We have performed shear-reversal experiments [21, 38] on the nonvibrated suspensions to evaluate the respective contributions of interparticle contacts and of hydrodynamic interactions to their shear viscosity. The principle of these experiments is the following: (i) the suspension is first sheared at a given shear stress or shear rate in a simple shear flow until steady-state is reached; (ii) the direction of shear is subsequently reversed (keeping the same value of the applied shear rate or stress), and the viscosity evolution with strain is recorded. The underlying idea is that interparticle contacts switch from compression to traction and are suddenly broken upon shear reversal. This usually results in an abrupt decrease of the viscosity, the minimum of which can be associated mostly with hydrodynamic interactions [38].

In order to have the best possible time, stress and strain resolution, as required by such experiments, we have used here a Kinexus Ultra+ rheometer (Netzsch). A similar geometry $(25 \mathrm{~mm}$ dimameter six blades vane in a $50 \mathrm{~mm}$ diameter cup) is used as in the vibration experiments to ensure accurate quantitative comparison of the results obtained in both kind of experiments. Since we use a stress-controlled rheometer, we work here in the controlled-stress mode in order to monitor accurately the transient evolution of the viscosity with strain. As in [39], we first shear the suspension at a constant imposed shear rate $\dot{\gamma}$ until the shear strain $\gamma$ exceeds 10 and a steady state of stress $\sigma$ is reached; this step is necessary to capture the initial plastic behavior. We then switch to stress-controlled mode and apply the measured steady-state stress $\sigma$ during $5 \mathrm{~s}$. We then apply a resting period of $10 \mathrm{~s}$ at zero stress before applying a stress $-\sigma$ until a new steady state is reached. We monitor the evolution of the shear viscosity both before and after shear reversal. These measurements are repeated for several values of $\dot{\gamma}$ (and thus of $\sigma$ ).

\section{EXPERIMENTAL RESULTS}

In this section, we first discuss in detail the behavior of a $29 \mathrm{wt} \%$ suspension and its origin. $29 \mathrm{wt} \%$ is the maximum particle fraction that we have investigated, close to the random close packing value $\phi_{\mathrm{rcp}}^{\mathrm{wt}} \simeq 0.3$. As such, it allows us to highlight the impact of vibrations on jamming. After presenting the rheological behaviour of the $29 \mathrm{wt} \%$ suspension in the absence of vibrations, we show the influence of vibrations on its apparent viscosity and the impact of a change in the injected energy. We subsequently study the impact of a change of the particle fraction $\phi^{\mathrm{wt}}$ in the range $\phi_{m}^{\mathrm{wt}} \simeq 0.24<\phi^{\mathrm{wt}}<\phi_{\mathrm{rcp}}^{\mathrm{wt}} \simeq 0.3$. We finally compare the short time response to shear-reversal and the steady-state response to vibrations.

\section{A. Non-vibrated suspensions: plastic jammed materials}

Fig. 5 displays the flow curve of the non-vibrated 29 wt $\%$ suspension measured with a shear-rate ramp.
In the absence of vibrations, the suspension behaves as a yield stress fluid: no steady flow is observed below a yield stress $\sigma_{y} \simeq 300 \mathrm{~Pa}$. The behavior is mostly plastic, with negligible viscous effects in the range of shear rates $\dot{\gamma}$ investigated. This leads to an apparent viscosity $\eta(\dot{\gamma}) \simeq \sigma_{y} / \dot{\gamma}$, thus decreasing with the shear rate with a power law close to -1 (this is evidenced in Fig. 5a). Note that fracture and progressive digging of the free surface are observed, which is classical with plastic materials; the observed $\sigma_{y}$ is thus only an apparent value. The progressive digging results in the initial decrease of the shear stress when increasing the shear rate in Fig. 5b. A more accurate characterization of the plastic behavior is presented in the appendix B.

The observation of such a plastic plateau is reminiscent of that found in unvibrated dry [34, 40] and wet [25, 41] granular materials in Couette geometries. This suggests a purely frictional origin of the observed behavior.

The main difference between our observations and those mentioned above is that dilation was free to occur in the vertical (vorticity) direction in the previous works, whereas the particle assembly is confined by the liquid interface surface tension in our case. When sheared, as $\phi>\phi_{m}=\phi_{c}$, the steady flow of the material implies dilation. This leads the particles to protrude from the liquid-air interface $[5,42]$ in our material, as we observe experimentally: the free surface, smooth and shiny at rest, becomes rough and scatters light upon shear. It can thus be assumed that a pressure is applied by capillarity on the particles at the free surface, which reaches at steady-state its maximum possible value $p_{\text {cap }} \propto \Gamma_{t} / r_{p}$, where $\Gamma_{t}$ is the liquid/air surface tension and $r_{p}$ is the mean particle radius. In our case $p_{\text {cap }} \simeq 5000 \mathrm{~Pa}$, whereas $p_{\text {cap }}=0$ in $[25,41]$ due to the presence of supernatant.

We note that dilation at the free surface is limited, and also necessarily occurs in the radial direction. The flow imposed by the rotation of the vane tool implies that volume fraction decreases down to a local value $\leq \phi_{m}$ close to the tool in a thin flowing shear band, the rest of the material being jammed [43] and seeing its volume fraction increase to ensure that the average volume fraction remains unchanged. The values of shear rate provided in this frictional state are thus only apparent values.

The total particle pressure applied on the granular assembly can be decomposed as $p=p_{\text {cap }}+p_{\text {grav }}$ where the pressure due to gravity depends on the depth $z$ (whereas $p_{\text {cap }}$ does not) and is $p_{\text {grav }}=\phi \Delta \rho g z$. $p_{\text {grav }}$ reaches a maximum value $\simeq 50 \mathrm{~Pa}$ at the bottom of the vane tool in our material, which is much less than $p_{\text {cap }}$. Then, as discussed in the introduction, the particle pressure being fixed by its bound $p_{\text {cap }}$, the shear stress is $\sigma=\mu\left(I_{v}\right) p$. The friction coefficient $\mu\left(I_{v}\right)$ a priori depends on the viscous number $I_{v}=\eta_{f} \dot{\gamma} / p$ [7]. Here, $I_{v}$ ranges between $10^{-8}$ and $10^{-5}$, which is in the range where $\mu\left(I_{v}\right)$ is observed to stay constant in the literature $[3,7]$. This explains the observation of a plastic plateau $\sigma_{y}=\mu\left(I_{v}=0\right) p$ in Fig. 5b. The major role of $p_{\text {cap }}$ in our experiments can be shown by studying the value of the torque plateau as a function of the immersed height $H$ of the vane: as the gravity stress varies with depth, the resulting shear stress $\mu p_{\text {grav }}$ leads to a $\propto H^{2}$ contribution to the torque, whereas the shear stress $\mu p_{\text {cap }}$ resulting 

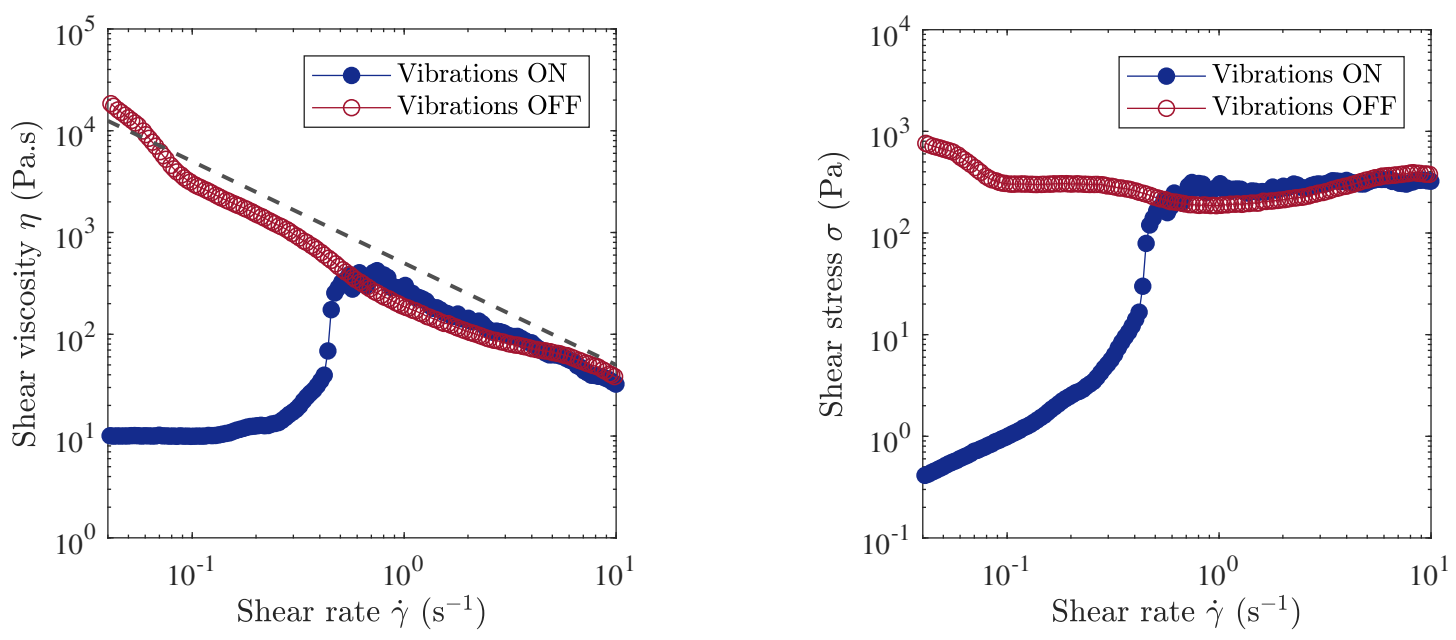

FIG. 5. Flow curve of a $29 \mathrm{wt} \%$ silica suspension with (filled circles, blue) and without (empty circles, red) vibrations: (a) shear viscosity $\eta$ vs. shear rate $\dot{\gamma}$, (b) shear stress $\sigma$ vs. $\dot{\gamma}$. The amplitude and frequency of vibrations are $500 \mu \mathrm{m}$ and $20 \mathrm{~Hz}$ respectively. A dashed line $\eta \propto 1 / \dot{\gamma}$ is shown in Fig. 5a to better evidence the plastic behavior of the non-vibrated suspension.

from the capillary pressure is constant and leads to a $\propto H$ contribution to the torque. This is studied and discussed in detail in the appendix B: here we find $T \propto H$, which confirms that the particle pressure is dominated by capillarity, as expected from the relative estimated values of $p_{\text {cap }}$ and $p_{\text {grav }}$. Finally, as discussed in detail in the appendix $\mathrm{B}$, the material friction coefficient can be roughly estimated as $\mu\left(I_{v}=0\right) \simeq 0.12$, which is close to values found in the quasistatic regime of dry granular materials [9].

\section{B. Vibrated suspensions: shear-thickening viscous materials}

We now discuss the differences between a vibrated suspension and a non-vibrated one. Fig. 5 displays the flow curve of a vibrated $29 \mathrm{wt} \%$ suspension, for an amplitude of $500 \mu \mathrm{m}$ and frequency of $20 \mathrm{~Hz}$, on the same graphs as those for the non-vibrated one.

When vibrations are applied, as Hanotin et al. [25], we observe that the material has no more yield stress. Instead, a liquid-like behavior is observed with a pseudo-Newtonian plateau at low shear rate, of viscosity around $\eta=10 \mathrm{~Pa}$.s. As the shear rate increases, shear-thickening is observed: the viscosity abruptly jumps by an order of magnitude at a critical shear rate of order $0.5 \mathrm{~s}^{-1}$ (critical shear stress of order $5 \mathrm{~Pa}$ ). At larger rates, the plastic behavior of the non-vibrated suspension is recovered with an apparent viscosity $\eta \propto 1 / \dot{\gamma}$.

We checked that the impact of vibrations on a given sample is reversible: when turning alternatively vibrations on and off at a given low $\dot{\gamma}$, the material behavior switches reversibly form the low viscosity plateau (blue curve in Fig. 5) to the high stress plateau (red curve in Fig. 5). We also recall (Sec. II A) that we checked that the particles are not broken during the experiments: their size is neither affected by flow nor by vibrations.

The two regimes might be understood as follows: low $\dot{\gamma}$ : at low shear rate $\dot{\gamma}$, the vanishing yield stress and the observation of a viscous regime means that the jamming volume fraction $\phi_{j}$ of the suspension, initially equal to $\phi_{m}$ and smaller than the material volume fraction $\phi$, has been increased above $\phi$ under the action of vibrations. This implies that contacts between particles are, at least partially, constantly broken by the vibrations during the flows, as proposed by Hanotin et al. [25]. $\phi_{j}$ can a priori tend towards $\phi_{\text {rcp }}$ if no contact is able to persist under the action of vibrations: the suspension would then behave as a suspension of frictionless particles. This possibility is discussed in Secs. III E and IV.

high $\dot{\gamma}$ : when increasing $\dot{\gamma}$, at a critical shear rate $\dot{\gamma}_{c} \simeq 0.5 \mathrm{~s}^{-1}$ (critical shear stress $\sigma_{c} \simeq 5 \mathrm{~Pa}$ ), the material suddenly behaves as a yield stress fluid again, i.e., shear jamming occurs. This means that the jamming volume fraction $\phi_{j}$ is decreased below $\phi$, likely back to its initial value $\phi_{m}$ for the frictional suspension. This transition between two regimes points to a competition between contact disruption by vibrations and contact formation by shear: there is a critical value of stress or shear rate for which shear-induced contact formation starts to be more efficient. The appropriate criterion is discussed in Secs. IIIC and IV. Note that in the shear-jammed regime, as for the non-vibrated suspension, flow must result in the separation of the system into a low-density-flowing band (of local volume fraction $\leq \phi_{m}$ ) close to the vane tool and a highdensity-jammed region; the reported shear rate value is thus an apparent value.

It is worth noting that thanks to the use of a vane tool, the whole material bulk is vibrated. If a bob had been used instead, a secondary orthogonal shear flow would have been generated [44]; such 3D flow would have led to an apparent fluidization for dominant orthogonal shear flows, as ob- 


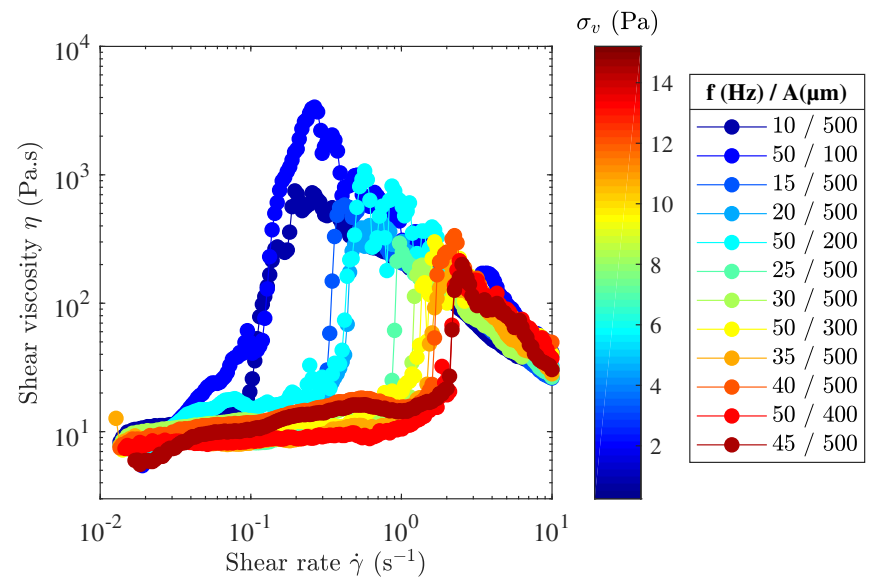

FIG. 6. Flow curves of a vibrated $29 \mathrm{wt} \%$ silica suspension: shear viscosity $\eta$ vs. shear rate $\dot{\gamma}$ for different values of the amplitude $A=[100-500] \mu \mathrm{m}$ and frequency $f=[10-50] \mathrm{Hz}$ of vibration. The legend also shows the values of the corresponding vibration stress $\sigma_{v}$.

served for superimposed flows of yield stress fluids [45, 46]. Here, as in Hanotin et al. [25], the impact of vibration can be fully attributed to vibration-induced microstructural changes, as shown by Gaudel et al. [28].

\section{Effect of the vibration stress $\sigma_{v}$}

To further understand the role of vibrations, we now study the impact of a change of the amplitude $A$ and frequency $f$ of vibration on the observed behavior. Fig. 6 displays the flow curve of a vibrated $29 \mathrm{wt} \%$ suspension, for various values of $A$ and $f$.

The same behavior is observed as in Sec. III B in all the studied cases, that is, a fluidization at low shear rate and a shear-jamming transition at a critical shear rate $\dot{\gamma}_{c}$. The main feature observed here is a significant increase of $\dot{\gamma}_{c}$ with both $A$ and $f$; by contrast, a change of $A$ and $f$ does not seem to have a significant impact on the viscosity of the viscous and shearjammed states (such possible impact is discussed below).

According to Marchal, Smirani, and Choplin [34] and Hanotin et al. [25], the relevant parameter tuning the material behavior is the density of vibration energy (Eq. (1)), recalled here: $\sigma_{v}=1 / 2 \rho_{s} A^{2}(2 \pi f)^{2}$. Dimensionally, $\sigma_{v}$ is also a stress characteristic of the vibrations. In Fig. 6, it is observed that, indeed, $\sigma_{v}$ allows to rationalize the data: when varying independently or simultaneously $A$ and $f$, shear jamming is observed at higher $\dot{\gamma}_{c}$ when $\sigma_{v}$ is increased.

To further test this idea, we plot the critical shear rate $\dot{\gamma}_{c}$ and the critical shear stress $\sigma_{c}$ as a function of $\sigma_{v}$ in Fig. 7. The $\dot{\gamma}_{c}$ values were obtained by choosing the last data point before the viscosity jump on the flow curves of Fig. 6. For $\sigma_{c}$, since we work at controlled shear rate, there is a huge stress variation at the shear-jamming transition and the exact onset stress is hard to determine directly; we thus chose to estimate the value of $\sigma_{c}$ by multiplying the value of the average over

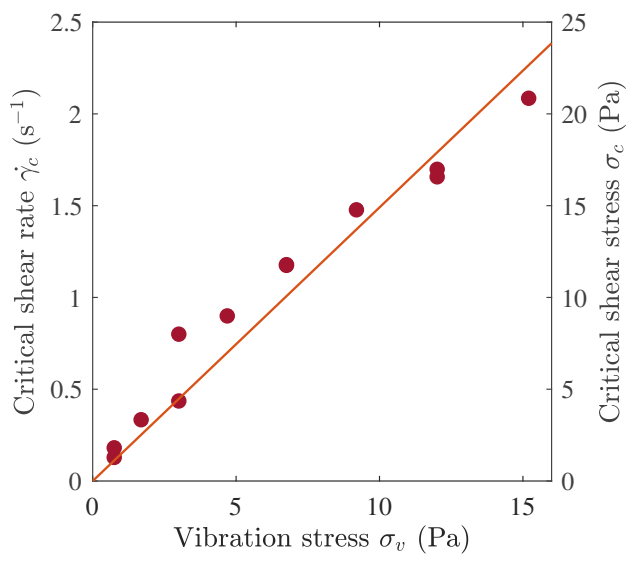

FIG. 7. Critical shear rate $\dot{\gamma}_{c}$ and critical shear stress $\sigma_{c}$ for shear jamming in the $29 \mathrm{wt} \%$ suspension as a function of $\sigma_{v}$. The lines is linear fits to the data. We find $\sigma_{c} \simeq 1.5 \sigma_{v}$.

all experiments of the viscosity plateau observed the low shear rate regime (here, 10 Pa.s) with the critical shear rate $\dot{\gamma}_{c}$.

We observe in Fig. 7 that $\dot{\gamma}_{c}$ and $\sigma_{c}$ seem to be linearly proportional to $\sigma_{v}$. This proportionality with both $A^{2}$ and $f^{2}$ is also clear when they are plotted versus $A$ for a given value of $f$, and versus $f$ for a given value of $A$; these plots are shown in the appendix $\mathrm{C}$.

We also note that the viscosity plateau in the fluidized suspension does not vary much in the range of investigated $\sigma_{v}$. This feature differs from the observations of Hanotin et al. [25] who found this viscosity to be clearly $\propto 1 / \sqrt{\sigma_{v}}$. This dependence was attributed to the increased fraction of open contacts with $\sigma_{v}$ [26]. Our observations might thus imply that the vibrations are fully efficient to open all contacts at low shear rate in our case. This would need to be investigated in more detail. In particular, does critical values of $\sigma_{v}$ exist for initiating fluidization and for reaching full fluidization? This cannot be investigated with our set-up due to the technical limits of the vibrating device $\left(1 \mathrm{~Pa} \leq \sigma_{v} \leq 15 \mathrm{~Pa}\right)$.

\section{Effect of the particle fraction}

We now study the impact of a change of the particle fraction on the flow curves. Data have been collected for different mass fractions $\phi_{m}^{\mathrm{wt}}<\phi^{\mathrm{wt}}<\phi_{\mathrm{rcp}}^{\mathrm{wt}}$ for various amplitudes and frequencies of vibrations. The flow curves obtained in the absence of vibration and at a given amplitude $A=500 \mu \mathrm{m}$ and frequency $f=20 \mathrm{~Hz}$ of vibration (corresponding to a vibration stress $\sigma_{v}=3 \mathrm{~Pa}$ ) for the different studied mass fractions are displayed in Fig. 8. The results obtained for a $28 \mathrm{wt} \%$ and a $26 \mathrm{wt} \%$ suspension with different vibration stresses are also shown in the appendix D.

In the absence of vibrations, all materials display a yield stress fluid behavior, as expected since $\phi_{m}^{\mathrm{wt}}<\phi^{\mathrm{wt}}$. This leads to an apparent shear-thinning behavior with $\eta \propto 1 / \dot{\gamma}$ at low $\dot{\gamma}$. The observed yield stress decreases as $\phi^{\text {wt }}$ is decreased. Given the origin of the yield stress discussed in Sec. III A, this is likely due to the fact that the local suspension dilation re- 

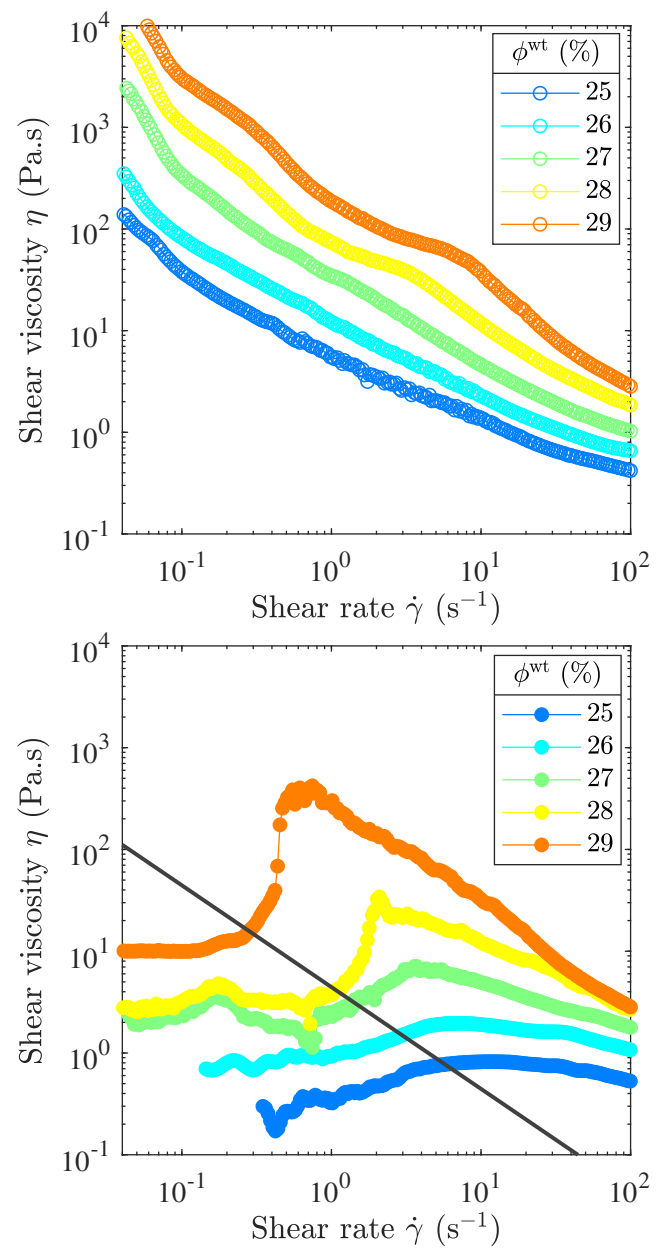

FIG. 8. Flow curves of the silica suspensions at different mass fractions. Top: without vibration. Bottom: for a given amplitude $A=500 \mu \mathrm{m}$ and frequency $f=20 \mathrm{~Hz}$ of vibration, which corresponds to $\sigma_{v}=3 \mathrm{~Pa}$; the solid line is a constant stress $\sigma_{c}=4.5 \mathrm{~Pa}$ line.

quired by flow decreases as $\phi^{\mathrm{wt}}$ gets closer to the critical state fraction $\phi_{c}^{\mathrm{wt}}=\phi_{m}^{\mathrm{wt}} \simeq 0.24$. This leads to a decreased particle protrusion at the particle interface and to a decrease of $p_{\text {cap }}$, and finally to a decrease of $\sigma_{y}=\mu\left(I_{v}=0\right) p_{\text {cap }}$. Predicting the $\phi^{\mathrm{wt}}$ dependence of $\sigma_{y}$ is beyond the scope of our study.

In the presence of vibrations, for all investigated $\phi^{\mathrm{wt}}$, the yield stress vanishes to give rise to a liquid-like pseudoNewtonian behavior at low shear rate. The flow curves then present a shear-thickening transition at high shear rate, where the behavior in the absence of vibrations is recovered. As classically observed for shear-thickening suspensions [4], the overall viscosity increases as the concentration $\phi^{\mathrm{wt}}$ is increased, and shear thickening is observed at lower and lower $\dot{\gamma}$ values as $\phi^{\mathrm{wt}}$ is increased. Moreover, the thickening transition is more and more abrupt as $\phi^{\mathrm{wt}}$ is increased: it seems to switch from continuous to discontinuous at the approach of $\phi_{\mathrm{rcp}}^{\mathrm{wt}}$.

It is often reported that shear-thickening occurs at a roughly constant shear stress when changing the particle fraction [4]; this is in reasonable agreement with our data, as highlighted

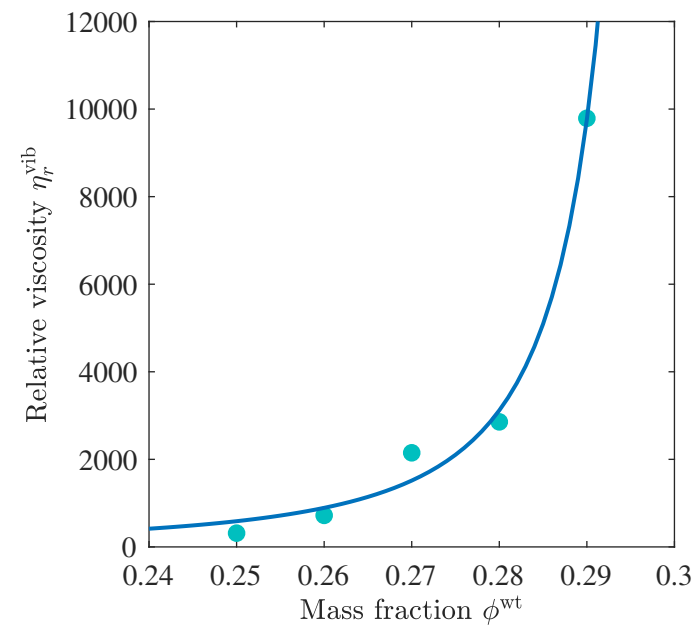

FIG. 9. Relative viscosity $\eta_{r}^{\mathrm{vib}}=\eta^{\mathrm{vib}}\left(\phi^{\mathrm{wt}}\right) / \eta_{f}$ in the liquid-like regime of vibrated suspensions as a function of the suspension mass fraction $\phi^{\mathrm{wt}}$. The line is a fit to a Maron-Pierce power law $\eta_{r}^{\mathrm{vib}}=$ $C\left(1-\phi^{\mathrm{wt}} / \phi_{j}^{\mathrm{wt}}\right)^{-2}$, with $C=18$ and $\phi_{j}^{\mathrm{wt}}=0.303$.

by the constant shear stress line in Fig. 8. Consistently, when changing the vibration stress $\sigma_{v}$, the same behavior is observed for all $\phi^{\mathrm{wt}}$ as for the $29 \mathrm{wt} \%$ suspension, with a critical shear rate $\dot{\gamma}_{c}$ and a critical shear stress $\sigma_{c}$ increasing with $\sigma_{v}$ (see appendix D). $\sigma_{c}$ can finally be estimated as $\sigma_{c} \simeq 1.5 \sigma_{v}$ for all mass fractions, as was found for the $29 \mathrm{wt} \%$ suspension.

We now plot the relative viscosity $\eta_{r}^{\mathrm{vib}}=\eta^{\mathrm{vib}}\left(\phi^{\mathrm{wt}}\right) / \eta_{f}$ in the liquid-like regime of vibrated suspensions as a function of the suspension mass fraction $\phi^{\mathrm{wt}}$ in Fig. 9. At a given $\phi^{\mathrm{wt}}$, we evaluate the viscosity value $\eta^{\mathrm{vib}}\left(\phi^{\mathrm{wt}}\right)$ at low shear stress $\sigma=$ $0.1 \mathrm{~Pa}$ for a vibration stress $\sigma_{v} \simeq 3 \mathrm{~Pa}$. The viscosity diverges with an asymptotic behavior consistent with a Maron-Pierce power law $\eta_{r}^{\mathrm{vib}}=C\left(1-\phi^{\mathrm{wt}} / \phi_{j}^{\mathrm{wt}}\right)^{-2}$, as classically observed in viscous suspensions [3], with $C=18$ and $\phi_{j}^{\mathrm{wt}}=0.303$. This jamming volume fraction $\phi_{j}^{\mathrm{wt}}$ is close to the estimated random close packing $\phi_{\mathrm{rcp}}^{\mathrm{wt}} \simeq 0.3$, which is the value expected for a frictionless suspension. This strongly suggests that the impact of vibrations is to open the particle contacts and that they are here fully efficient to open all contacts, creating a frictionless suspension. This possibility is investigated in the next section.

\section{E. Shear-reversal experiments:} Viscosity of frictionless suspensions

As vibrations are suspected to play a role on the particle contact network, we have performed shear-reversal experiments $[21,38]$ to disentangle the contributions of interparticle contacts and of hydrodynamic interactions to the shear viscosity of nonvibrated suspensions, as detailed in Sec. II B 2. Note that it is not possible to analyze such experiments for the $29 \mathrm{wt} \%$ suspensions as the material is fractured when sheared.

In Fig. 10, we plot the shear viscosity as a function of time before and after shear reversal for a $26 \mathrm{wt} \%$ suspension. 


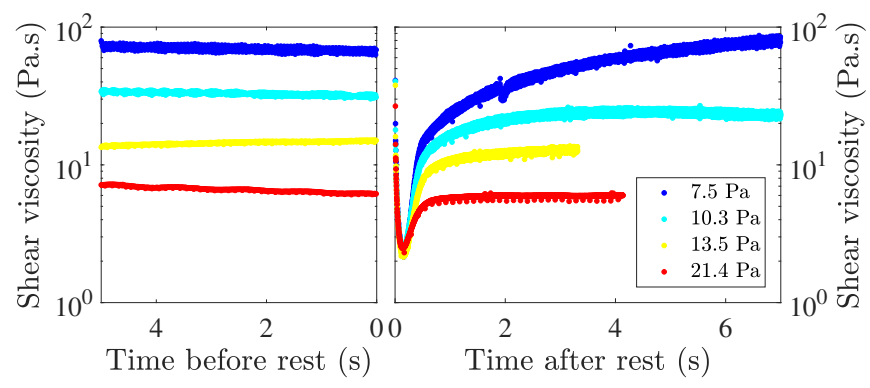

FIG. 10. Response to shear-reversal experiments of a $26 \mathrm{wt} \%$ suspension. Different stresses are applied (see legend). Left: Steady-state viscosity as a function of the time before rest and reversal. Right: Evolution of the viscosity as a function of the time elapsed since the beginning of reversal.

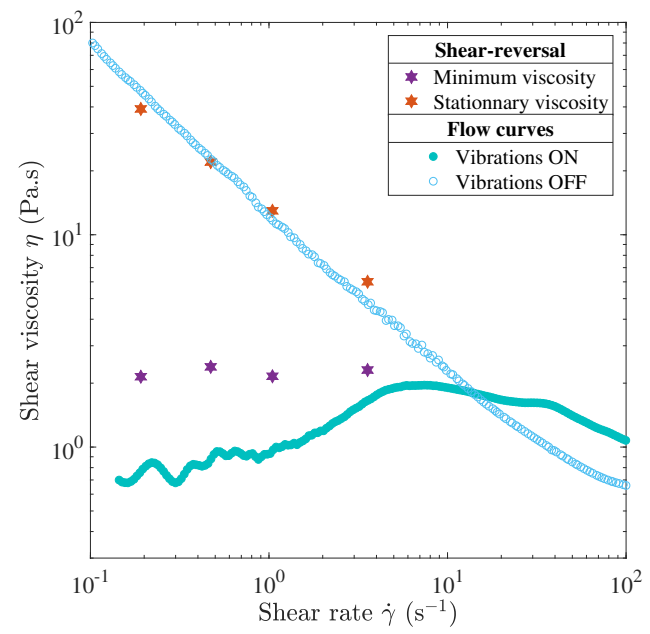

FIG. 11. Shear viscosity versus shear rate for a $26 \mathrm{wt} \%$ suspension. Stars: steady-state and minimum viscosity obtained from shearreversal experiments. Circles: flow curves obtained from a shear rate ramp with (filled circles) or without (empty circles) vibrations; here $\sigma_{v}=3 \mathrm{~Pa}$.

As classically observed in concentrated suspensions [21, 38], there is a drop of the shear viscosity upon shear reversal for each applied stress (the same is observed for all studied mass fractions); it subsequently increases with time until it reaches the same steady state as in the reverse direction for a strain of order 5. In the range of stresses investigated, the viscosity drops by a factor 2 to 50 .

The minimum viscosity $\eta_{\min }$ achieved during the reversal is plotted versus the applied shear stress in Fig. 11 for the $26 \mathrm{wt} \%$ suspension, together with the steady state viscosity; the flow curves obtained from a shear rate ramp with or without vibrations are also displayed. Similar curves are shown for the other investigated mass fractions in the appendix E.

Strikingly, $\eta_{\min }$ is quasi independent of the applied shear stress for each particle fraction. This is consistent with the results of Peters et al. [38] who showed that $\eta_{\min }$ value is close to the pure hydrodynamic contribution to the suspension viscosity. Although the microstructure of frictional and frictionless suspensions are expected to present some differences, $\eta_{\text {min }}$ can thus be seen as a good estimate of the viscosity of the suspension for frictionless particles, as was shown by Lin et al. [21].

When compared with the steady-state viscous behavior achieved under vibration at low stresses, it is observed that the $\eta_{\min }$ values are close to the viscosity of the vibrated sample for all investigated particle fractions. This supports the interpretation that vibrations tend to pull particles apart and to generate a suspension of frictionless particles. The results shown in the appendix $\mathrm{E}$ for other particle fractions lead to the same conclusion.

\section{DISCUSSION}

\section{A. Summary and scenario}

In this work, we have studied a suspension of volume fraction $\phi_{m}<\phi<\phi_{\text {rcp }}$. We recall that $\phi_{m}$ is the jamming volume fraction $\phi_{j}\left(\mu_{p}=\mu_{0}\right)$ for the suspension of particles of interparticle friction coefficient $\mu_{0} \neq 0$, which is also the volume fraction $\phi_{c}$ of the critical state of granular materials. In practice, it is the volume fraction at which the viscosity of the suspension diverges, and at which granulation starts. $\phi_{j}\left(\mu_{p}=0\right)$ is the jamming volume fraction of the same particles in the absence of frictional forces, as if the particles were frictionless; $\phi_{j}\left(\mu_{p}=0\right)$ is equal to the random close packing $\phi_{\text {rcp }}$ of the particle assembly.

In the absence of vibrations, consistent with $\phi_{m}<\phi$, we have observed that the suspension is jammed. Thus, it behaves as a dilatant granular material, and flows only above a yield stress $\sigma_{y}$. Here, the suspension is confined by the liquid interface surface tension, with a confinement pressure $p$, and $\sigma=\sigma_{y} \simeq \mu(0) p$ for any imposed shear rate, where $\mu(0)$ is the friction coefficient of the granular assembly in the limit of vanishing viscous number $I_{v}$, as all of our data are obtained for $I_{v} \leq 10^{-5}$. The confinement pressure $p$ depends on $\phi$ : it is bounded by a maximum value $p_{\text {cap }}^{\text {cap }} \sim \Gamma_{t} / r_{p}$, which seems to be achieved only at the highest investigated particle fraction.

Under vibrations, we have observed fluidization of the suspension at low shear rate/shear stress in the whole range of amplitude and frequency of vibration investigated. This implies that the suspension jamming fraction $\phi_{j}$ is affected by the vibrations and increases above $\phi$. As in [26], this is attributed to vibration-induced contact disruption. We note however that the viscosity in this regime seems to be independent of $\sigma_{v}$ by contrast with [25], which would imply that the efficiency of contact disruption by vibrations is here maximal. Consistently, this low shear rate viscosity is similar to the minimum viscosity observed in shear-reversal experiments, and it increases with $\phi$ as expected for a frictionless suspension, with a Maron-Pierce law diverging at RCP.

A transition to shear jamming has then been observed at a critical shear rate/shear stress. In the shear-jammed regime, the material seems to recover the behavior observed in the absence of vibrations. This points to a transition between a frictional and a frictionless suspension when turning vibrations on, and between a frictionless and a frictional suspension 


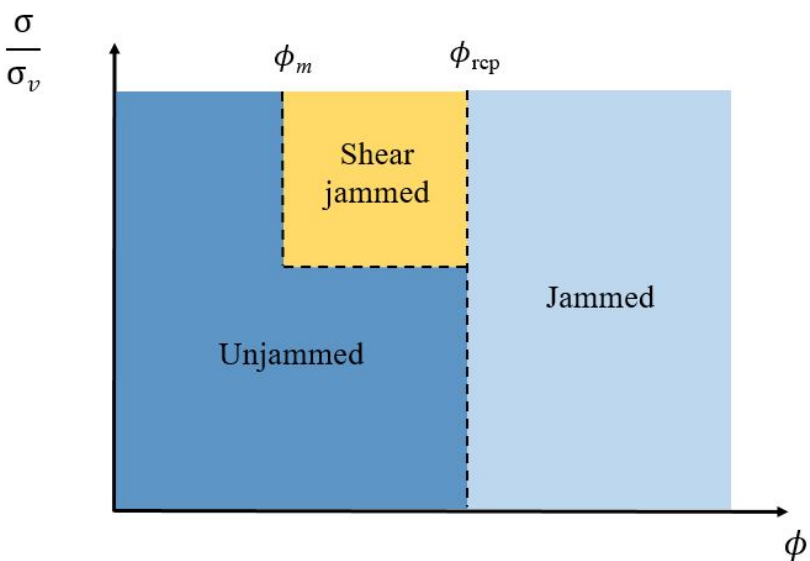

FIG. 12. Jamming diagram of the suspension in the space of dimensionnless stress $\sigma / \sigma_{v}$ and volume fraction $\phi$.

when increasing the shear rate/shear stress on the vibrated suspension. In other words the jamming volume fraction would change from $\phi_{j}\left(\mu_{p}=\mu_{0}\right)=\phi_{m}<\phi$ to $\phi<\phi_{j}\left(\mu_{p}=0\right)=\phi_{\text {rcp }}$ when turning vibrations on at low shear rate, and then from $\phi<\phi_{j}\left(\mu_{p}=0\right)=\phi_{\text {rcp }}$ to $\phi_{j}\left(\mu_{p}=\mu_{0}\right)=\phi_{m}<\phi$ when increasing the shear rate under vibrations.

The scenario of a competition between contact disruption by vibrations and contact formation by shear is similar to the mechanism proposed by Hanotin et al. [25]; however, they did not observe shear jamming. This striking difference is likely due to the fact that they worked with a settled suspension, free to dilate in the vorticity direction, whereas we work with a confined suspension at constant volume fraction $\phi$. Indeed, in Hanotin et al. [25], a constant confining stress (gravity) was applied on a settled suspension; the volume fraction then resulted from a complex interplay between gravity, vibrations, and shear, but was free to vary, likely preventing shear jamming to occur. When increasing $\dot{\gamma}$, it is likely that $\phi$ decreases simultaneously to $\phi_{j}$ in the work of Hanotin et al. [25], i.e., $\phi$ may remain below $\phi_{j}$ even when vibrations are no more efficient to break contacts.

Our observations can be summarized in the schematic jamming diagram shown in Fig. 12. They might be understood in the framework of the model developed by Wyart and Cates [16] to model the behavior of shear-thickening suspensions, and by using the vibration stress $\sigma_{v}$ introduced by Hanotin et al. [25] to account for the impact of vibrations on the behavior of frictional suspensions, as discussed below.

\section{B. Shear thickening and the Wyart and Cates (2014) model}

In order to better understand our observations, it is necessary to come back to the recent developments in the literature of shear thickening.

Shear thickening, i.e., the increase of the apparent viscosity with the load, is observed in the absence of vibrations in most concentrated non-Brownian suspensions with particle size in the range $1 \sim 100 \mu \mathrm{m}$. It is more and more abrupt as the volume fraction is increased, and may become discontinuous, meaning that there is a jump in the viscosity under controlled shear rate at some critical value $[3,4]$. Strictly speaking, discontinuous shear thickening (DST) is the transition from a viscous state to a more viscous state with a finite value of viscosity and homogeneous flow. At the vicinity of the jamming transition, shear jamming is then observed: under controlled stress, the flow abruptly stops at some critical value, meaning that the apparent viscosity becomes infinite. In the case of shear jamming, no flow is possible above a critical shear rate. Experimentally, some confusion between DST and shear jamming can then be due to the fact that macroscopic flows can be observed at any controlled macroscopic shear rate, even if the material shear jams: in this latter case, the material becomes inhomogeneous and shear banding occurs as zones of lower density form to accommodate the boundary conditions [31]. As discussed previously, the thickening we observe under controlled shear-rate in our vibrated suspensions is shear jamming.

Recent theoretical and numerical studies of Wyart and Cates [16] and Mari et al. [17] have related shear thickening to a transition from lubricated (frictionless) to frictional contacts with the increase in stress. This has been linked to the existence of a short-range repulsive force between the particles and to the competition between this force and hydrodynamic forces. If such repulsive force exists, the contacts are lubricated (frictionless) at low shear rate, as the particles cannot approach each others. At high load, the hydrodynamic normal forces become sufficiently important to bring particles into contact as the repulsive forces are overcome. Interparticle friction then occurs, leading to an increase of the viscosity or to shear jamming; shear jamming is then the creation of a macroscopic jammed contact network induced by shear. Experimental evidence for this microscopic description of the shear-thickening transition has been recently presented by Comtet et al. [19] thanks to direct interparticle force measurements.

As shown by Wyart and Cates [16], the whole shear thickening behavior of model suspensions such as suspensions of non-Brownian spheres of volume fraction $\phi$ can be explicitly modeled with the help of three ingredients: (i) a stressdependent fraction of interparticle frictional contacts in the suspension $f_{c}(\sigma)$, the other contacts being lubricated; (ii) a $f_{c}$-dependent jamming volume fraction $\phi_{j}\left(f_{c}\right)$; and (iii) a $\phi_{j^{-}}$ dependent viscosity function $\eta_{r}\left(\phi / \phi_{j}\right) . f_{c}(\sigma)$ is a monotonically increasing function of $\sigma$ such that $f_{c}(0)=0$ and $f_{c}(\sigma \rightarrow$ $\infty)=1$. In the absence of contacts $\left(f_{c}=0\right), \phi_{j}(0)=\phi_{\mathrm{rcp}}$, whereas when all contacts are frictional $\left(f_{c}=1\right), \phi_{j}(1)=$ $\phi_{j}\left(\mu_{p}=\mu_{0}\right)=\phi_{m} ; \phi_{j}\left(f_{c}\right)$ is then a monotonically decreasing function of $f_{c}$ interpolating between these two values. Finally $\eta_{r}\left(\phi / \phi_{j}\right)$ is a monotonically increasing function of $\phi / \phi_{j}$ diverging at $\phi / \phi_{j}=1$. With these ingredients, if the suspension volume fraction $\phi$ is lower than $\phi_{j}\left(f_{c}=1\right)=\phi_{m}$ then DST can be observed in some conditions as a transition from a low viscosity state (of viscosity diverging at $\phi_{j}\left(f_{c}=0\right)=\phi_{\mathrm{rcp}}$ ) to a high viscosity state (of viscosity diverging at $\phi_{m}$ ). If $\phi_{m}<\phi<\phi_{\mathrm{rcp}}$, shear jamming is observed as no flow is possible without dilatancy for the frictional material at such value 
of $\phi$, whereas the frictionless material can flow. Explicit equations and a test of the model in its simplest form for our suspensions are given below and in the appendix F.

Experimental evidence for this macroscopic description of the shear-thickening transition has been provided by Guy, Hermes, and Poon [18]. They plotted the low-viscosity plateau (before shear thickening) and the high-viscosity plateau (after shear thickening) of a thickening granular suspension for different volume fractions. They showed that the lower plateau tends to diverge at $\phi \simeq 0.64=\phi_{\text {rcp }}$ corresponding to frictionless spheres, and that the higher plateau tends to diverge at the random loose packing $\phi \simeq 0.55=\phi_{\text {rlp }}$, as expected for highly frictional spheres.

\section{Modelling of shear jamming in vibrated suspensions}

In the Wyart and Cates [16] work, the variation of $f_{c}$ with the shear stress $\sigma$ comes from the existence of a short-range repulsive force $F_{r}$ between the particles. At rest and at low applied shear stress, there is then no direct contact between the particles; at high shear stress, the hydrodynamic normal forces between the particles can overcome the repulsive force to create frictional contacts. An empirical function describing the fraction of contacts, consistent with the above description, is then

$$
f_{c}=\exp \left(-\left(\sigma_{r} / \sigma\right)^{\beta}\right)
$$

where $\sigma_{r}$ is the typical repulsive stress to overcome and $\beta \simeq 1$ is a constant parameter. $\sigma_{r}$ was shown to be linked to the repulsive force $F_{r}$ in numerical simulations [12] as

$$
\sigma_{r}=2.9 F_{r} /\left(3 \pi d^{2}\right)
$$

for suspensions of particles of diameter $d$. Obviously, it is of the order of the critical stress $\sigma_{c}$ for shear thickening.

In our system, we have shown that there is no significant repulsion force. Vibrations have been shown, however, to act as an external source of energy that it dissipated through a constant diffusive motion of the particles [28]. The particle diffusion coefficient [28] has then been shown to be controlled by the kinetic energy density of vibrations $\sigma_{v}=1 / 2 \rho_{s} A^{2}(2 \pi f)^{2}$. Note however that Gaudel et al. [28] have studied this diffusive behavior only in the regime where the fluidized suspension viscosity also depends on $\sigma_{v}$ as in [27], which is not the case in our experiments; we cannot rely here on the diffusion coefficient scaling with $\sigma_{v}$ found by Gaudel et al. [28]. We can nevertheless consider that vibrations introduce an effective thermal-like repulsive force between the particles, which makes the particles frictionless as long as vibrations are efficient, as shown in Sec. III E.

This is reminiscent of the behavior of Brownian suspensions, which have been shown to display similar shearthickening behavior as suspensions of repulsive particles, the Brownian force playing the role of an additional repulsive force [47]. In the absence of a repulsive force, Mari et al. [47] found that the critical stress $\sigma_{c}$ of Brownian suspensions
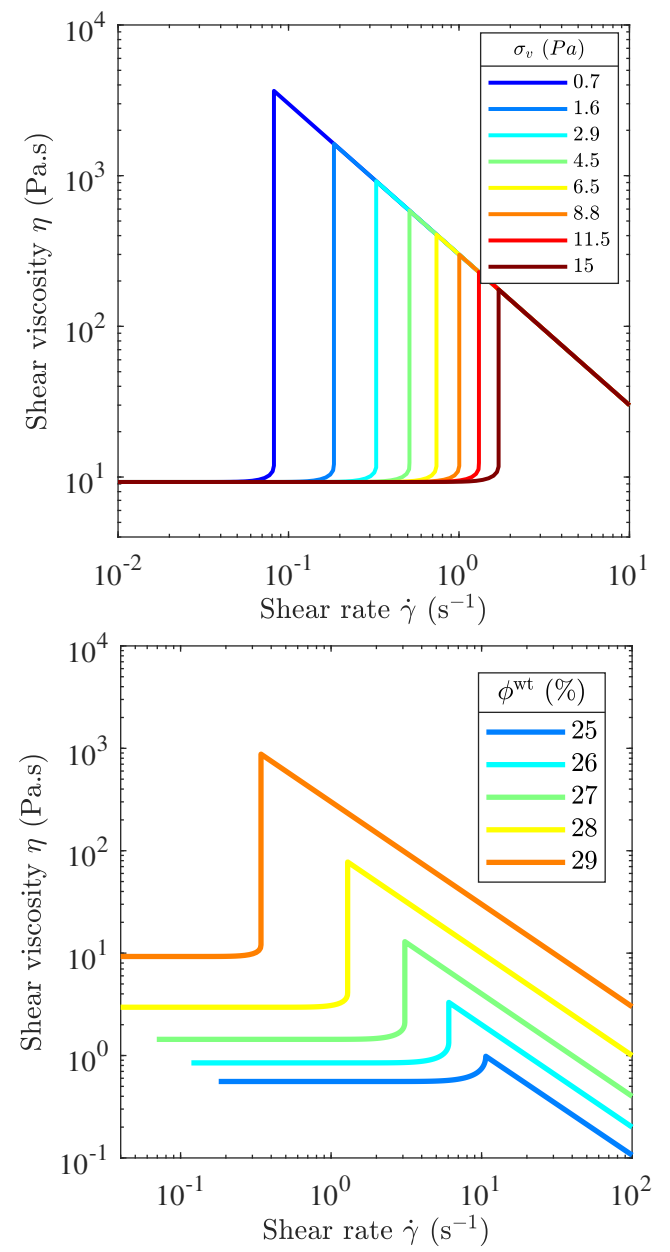

FIG. 13. Prediction of the Wyart and Cates [16] model for the viscosity vs. shear rate (see main text for the model parameters). Top: for $\phi^{\mathrm{wt}}=0.29$ for the various $\sigma_{v}$ investigated experimentally; bottom: for $\sigma_{v}=3 \mathrm{~Pa}$ for the various $\phi^{\mathrm{wt}}$ investigated.

at shear thickening is

$$
\sigma_{c} \simeq \frac{5 k_{B} T}{a^{3}}
$$

with $T$ the temperature, and $a$ the particle radius. In a Brownian suspension, the density of energy injected by the thermal bath is $\sim k_{B} T / a^{3}$, which is close to $\sigma_{c}$ for these suspensions. Identifying this density of energy and that injected by the vibrations, it thus seems natural to assume that the critical stress for shear thickening associated to vibrations is proportional to $\sigma_{v}$. This is precisely what we have observed experimentally $\left(\sigma_{c}=1.5 \sigma_{v}\right.$ in Fig. 7). Finally, it seems natural to use the approach of Wyart and Cates [16] described above to model our material shear-thickening, with Eq. (4) and a typical repulsive stress $\sigma_{r}$ of the order of the critical stress $\sigma_{c}$, i.e.,

$$
\sigma_{r}=\alpha \sigma_{v}
$$

where $\alpha$ is a constant expected to be of order 1 .

The model is detailed in the appendix F. Its predictions are shown in Fig. 13 for $\phi^{\mathrm{wt}}=0.29$ for the various $\sigma_{v}$ investigated experimentally (as in Fig. 6), as well as for $\sigma_{v}=3 \mathrm{~Pa}$ 
(corresponding to $f=20 \mathrm{~Hz}$ and $A=500 \mu \mathrm{m}$ for the $29 \mathrm{wt} \%$ suspension) for the various $\phi^{\mathrm{wt}}$ investigated (as in Fig. 8). For $\alpha=6$ in Eq. (7), the predicted variations of the behavior with both $\sigma_{v}$ and $\phi^{\mathrm{wt}}$ are fairly similar to those observed in Figs. 6 and 8. This shows the relevance of the proposed description of the impact of vibrations on the particles.

\section{CONCLUSIONS}

We have studied dense granular suspensions at high volume fraction, above the jamming volume fraction, but below the particle assembly random close packing. In the absence of vibrations, the suspensions have a solid-like behavior with a yield stress. Particles are here confined by the liquid interface and the yield stress is of frictional origin. When vibrations are applied, the yield stress disappears to give rise to a liquid-like pseudo-Newtonian behavior at low shear rate. As the shear rate is increased, we observe a shear-jamming transition: the apparent yield stress behavior is then recovered. We have shown that the observed shear-jamming transition can be tuned by changing the energy of vibrations injected into the system. In the framework of the Wyart and Cates (2014) model, vibrations can finally be seen as introducing a thermallike repulsive force, which yields a critical stress proportional to the vibration repulsive stress previously introduced by Hanotin et al. (2015). The vibrated suspensions then behaves as a frictionless suspension at low shear rate, as shown with shearreversal experiments, and the observed shear jamming can be interpreted as frictionless to frictional transition.

On the application side, vibrations are a promising route to unjam dense suspensions that are handled in the industry. This route is an alternative to macroscopic flow superposition [22, 23]. By evidencing the quantitative impact of the vibration stress $\sigma_{v}$ on the shear jamming transition, we provide the key elements to optimize the process and maximize the range of flowability.

On the fundamental side, vibrations seem to offer a promising way to investigate experimentally frictionless suspensions. There is finally an interesting analogy between the vibration stress in vibrated non-Brownian suspension and the Brownian stress in non-vibrated Brownian suspensions, which would deserve an in-depth investigation, in particular at the microscopic level.

\section{ACKNOWLEDGMENTS}

We acknowledge support from the Agence Nationale de la Recherche (project Fluididense ANR-17-CE07-0040), from Institut Pierre-Gilles de Gennes (laboratoire d'excellence, "Investissements d'avenir" program ANR-10-IDEX-0001-02 PSL and ANR-10-LABX-31 and ANR-10-EQPX-34), and from the European Funds Interreg VA GR (PowderReg project).

\section{Appendix A: Viscosity at low mass fraction}

In order to estimate the jamming fraction $\phi_{m}^{\mathrm{wt}}$ of the - frictional - suspensions, we have studied their flow behavior in their liquid-like regime $\left(0<\phi^{\mathrm{wt}}<0.24\right)$ with a Kinexus U1tra+ rheometer (Netzsch). Given the low viscosity of the suspending fluid $\left(\eta_{0}=9.5 .10^{-4} \mathrm{~Pa} . \mathrm{s}\right.$ at $\left.25^{\circ} \mathrm{C}\right)$, and thus of the suspension at low volume fraction, we have used a thin-gap bob-in-cup geometry (of inner radius $12.5 \mathrm{~mm}$, outer radius $13.75 \mathrm{~mm}$ and height $37.5 \mathrm{~mm}$ ) in these experiments, with serrated walls to avoid wall slip. Indeed, with the wide-gap vane-in-cup geometry used in the vibration experiments, momentum diffusion is long (of order $100 \mathrm{~s}$ ) at such viscosities and sedimentation might occur during the experiment. Constant stresses were applied until steady-state was reached; the time of the experiments was chosen as short as possible to avoid possible sedimentation. Data were collected only at low shear stress $\lesssim 0.1 \mathrm{~Pa}$ at the lowest studied particle fractions; indeed, inertial effects occurred at higher stresses at such low viscosities, leading to an apparent increase of the shear stress with the shear rate. Due to the limit of the rheometer, only data points for $\sigma \geq 0.01 \mathrm{~Pa}$ were recorded. The range in which the suspensions can be characterized at low volume fraction is thus very limited. The steady-state flow curves obtained within these conditions are shown in Fig. 14.

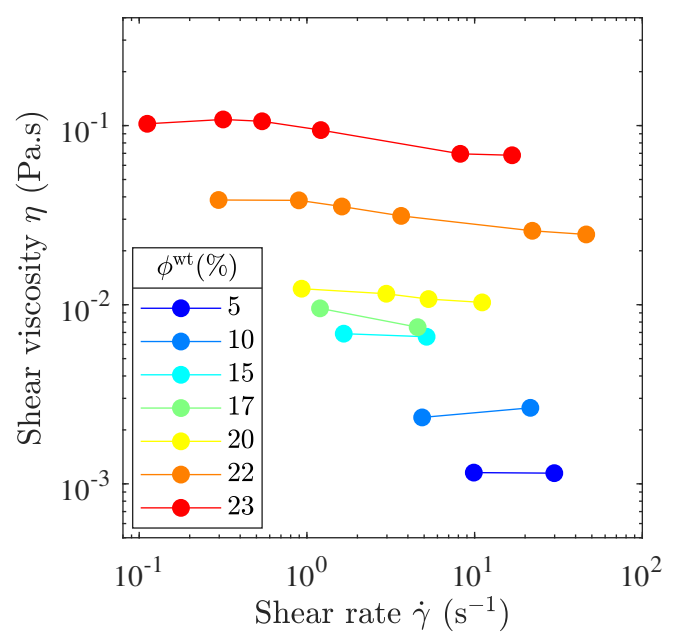

FIG. 14. Flow curves of the silica suspensions for mass fractions $0<\phi^{\mathrm{wt}}<0.24$ (see legend).

As expected, the overall viscosity increases with the mass fraction $\phi^{\mathrm{wt}}$. At low $\phi^{\mathrm{wt}}$, the behavior is consistent with a pseudo-Newtonian behavior. The sharp increase of the viscosity around $20 \mathrm{wt} \%$ is accompanied with a transition to a shearthinning behaviour. Shear thinning is commonly observed at high volume fraction in concentrated suspensions, and is likely linked to non-Coulomb or velocity-weakening friction coefficient between the particles [39, 48, 49]. The suspension relative viscosity $\eta_{r}\left(\phi^{\mathrm{wt}}\right)=\eta\left(\phi^{\mathrm{wt}}\right) / \eta_{0}$ is finally extracted from the data measured at a constant stress $\sigma=0.01 \mathrm{~Pa}$. The results are shown in Fig. 15. 


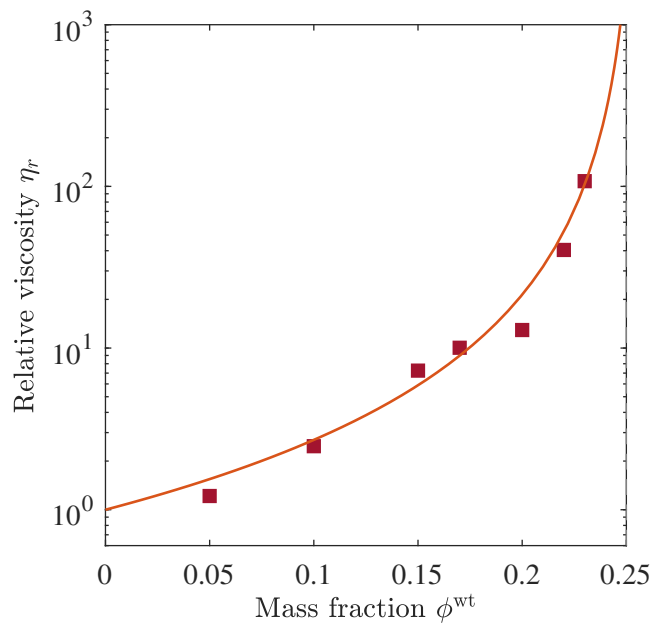

FIG. 15. Relative viscosity $\eta_{r}$ estimated at $\sigma=0.01 \mathrm{~Pa}$ as a function of the silica particle mass fraction $\phi^{\mathrm{wt}}$ (empty squares). The solid line is a fit of the data to the Maron-Pierce law $\eta_{r}=\left(1-\phi^{\mathrm{wt}} / \phi_{m}^{\mathrm{wt}}\right)^{-2}$ with $\phi_{m}^{\mathrm{wt}}=0.255$.

In order to estimate the jamming fraction of the suspension, we then fit the relative viscosity to a Maron-Pierce law $\eta_{r}=$ $\left(1-\phi^{\mathrm{wt}} / \phi_{m}^{\mathrm{wt}}\right)^{-2}$. Best fit is obtained for $\phi_{m}^{\mathrm{wt}}=0.255$; since, the $24 \mathrm{wt} \%$ suspension has a yield stress, this is of course only a rough estimate of the true jamming mass fraction of the frictional suspension. This estimate is consistent with the onset of granulation observed for non-vibrated suspensions in Sec. II A.

\section{Appendix B: Torque vs. height and yield stress origin}

As shown by Ancey and Coussot [41], for fluid-saturated non-buoyant frictional suspensions, the torque resisting slow flows in a Couette geometry is a quadratic function of the immersed height $H$ of the rotating tool. This contrasts with the linear function of $H$ found in the viscous regime of suspensions.

This is easily explained by recalling that the torque $T$ along the inner cylinder or the vane tool of radius $R$ and immersed height $H$ can be computed as

$$
T=2 \pi R^{2} \int_{z=0}^{z=H} \sigma(z) \mathrm{d} z
$$

and by considering the dependence with depth $z$ of the shear stress $\sigma(z)$.

For most materials, $\sigma(z)=\sigma_{0}$ does not depend on $z$ due to translational invariance and to independence of $\sigma$ with the (z-dependent) pressure. This then leads to $T=2 \pi R^{2} H \sigma_{0}$, i.e., $T \propto H$. For frictional materials (dry granular materials and granular suspensions), however, since $\sigma(z)=\mu p(z)$, where $\mu$ is the material friction coefficient and $p(z)$ is the particle pressure, $\sigma(z)$ might depend on $z$. This is the case for dry granular materials with a free top surface and for fluid-saturated non-buoyant granular suspensions. In this latter case, in the absence of a Janssen effect [35], the particle pressure is $p(z)=\phi \Delta \rho g z$, which leads to a torque $T=\pi R^{2} \mu \phi \Delta \rho g H^{2}$, i.e., $T \propto H^{2}$.

Nevertheless, even for a frictional material, one can find that $T \propto H$; this is found when a confining pressure larger than the gravity stress is directly applied on the grains, e.g., by a grid as in [7] or by the fluid interface surface tension when the particles protrude from the liquid/air interface due to dilation $[5,42]$. In that case, the total particle pressure applied on the granular assembly can be decomposed as $p(z)=p_{\text {cap }}+$ $p_{\text {grav }}(z)$ where the $z$-independent pressure due to capillarity at the top interface is $p_{\text {cap }}$ and the $z$-dependent pressure due to gravity is $p_{\text {grav }}$. If $p_{\text {cap }} \gg p_{\text {grav }}$, the material is characterized by a $z$-independent shear stress $\sigma_{0}=\mu p_{\text {cap }}$ and a torque $T \propto H$ is recovered.

For plastic flows with a vane tool, where flows are localized close to the tool, the bottom effects can easily be taken into account as:

$$
T=2 \pi \int_{r=0}^{r=R} \sigma^{\prime}(z=H) r^{2} \mathrm{~d} r=\frac{2 \pi R^{3}}{3} \sigma^{\prime}(z=H)
$$

In this equation, $\sigma^{\prime}(z=H)$ is the shear stress exerted at the bottom, that is, the $\sigma_{\theta z}$ component of the shear stress tensor, whereas the shear stress $\sigma$ exerted on the side of the vane tool is the component $\sigma_{r \theta}$. It results in an additional constant contribution to the torque $T$ in the case of $z$-independent stresses, and in a $T \propto H$ contribution in the case of gravity-induced $z$-dependent stresses.

In order to be more quantitative, the anisotropy of particle normal stresses can be considered. Denoting 1 the flow direction, 2 the gradient direction, and 3 the vorticity direction in a simple shear flow, it is know in dense suspensions [3] that the particle normal stresses are $\sigma_{11}^{p} \simeq \sigma_{22}^{p}$ and $\sigma_{33}^{p} \simeq 0.5 \sigma_{22}^{p}$; similar features are expected for granular materials. In the vane-in-cup geometry, the vorticity direction is that of gravity, i.e., $\sigma_{33}^{p}=\phi \Delta \rho g z$, and we measure the lateral shear stress $\sigma=\mu \sigma_{22}^{p} \simeq 2 \mu \sigma_{33}^{p}$. At the bottom, the shear stress is $\sigma^{\prime}=\mu \sigma_{33}^{p}$, that is, $\sigma^{\prime} \simeq \sigma / 2$. In the following, we will finally compare quasistatic measurements of the torque $T$ performed on granular suspensions to the two following expressions:

$$
T=\pi R^{2} \phi K \mu \Delta \rho g\left(H^{2}+\frac{2 R H}{3 K}\right)
$$

when the particle pressure is set by gravity, with $K=$ $\sigma_{22}^{p} / \sigma_{33}^{p} \simeq 2$, and

$$
T=2 \pi R^{2} \mu p_{\text {cap }}\left(H+\frac{R}{6}\right)
$$

when the capillary pressure $p_{\text {cap }}$ is dominant (since $p_{\text {cap }}$ is only an estimate of the particle normal stresses, it is not relevant to include $K$ in this last expression).

In order to show the relevance of this method to characterize the $z$-dependence of stresses and determine the value of $\mu$, we first show steady-state measurements performed on a dry granular material for various immersed heights $H$. The material is composed of monodisperse glass beads of diameter 
$125 \mu \mathrm{m}<d<150 \mu \mathrm{m}$. The granular material is poured in the cup (preparation does not impact steady-state flow), and the vane tool is immersed into the material at various heights $H$. The flow resistance is measured at steady-state in the quasistatic regime, for a macroscopic shear rate of $0.1 \mathrm{~s}^{-1}$. The results are shown in Fig. 16. As expected, the data follow a quadratic increase with $H$, and are well fitted to Eq. (B3). With $\Delta \rho \simeq 2500 \mathrm{~kg} \cdot \mathrm{m}^{-3}$ and an expected $\phi \simeq 0.6$ at the critical state [9], this yields $\mu \simeq 0.6$, which is in reasonable agreement with values reported at low inertial number for glass beads [9].

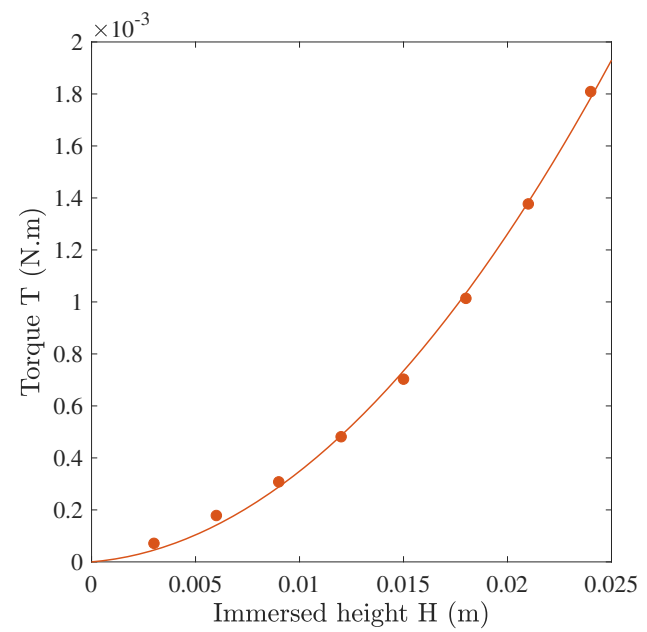

FIG. 16. Torque vs. immersed height for a dry granular material made up of monodisperse glass beads. The line is a fit of the data to Eq. (B3), with $\mu=0.62$.

In the following, we show steady-state measurements performed on settled silica suspensions, for various immersed heights $H$. A $22 \mathrm{wt} \%$ suspension is prepared in the cup and left at rest during 1 day to let full sedimentation occur. The vane tool is then immersed at various heights $H$ into the sediment, on top of which the supernatant is kept, and the flow resistance is measured at steady-state in the quasistatic regime, for a macroscopic shear rate of $0.1 \mathrm{~s}^{-1}$. The results are shown in Fig. 17.

As for the dry granular material, the torque data follow a quadratic increase with $H$; much lower values of $T$ are found, however, due to the presence of water and of buoyancy. This shows that there is no significant attraction between the silica particles, and that the particles mainly interact through frictional forces. In the absence of another confining pressure than gravity, the resulting resistance to flow is small, yielding an average shear stress of order $10 \mathrm{~Pa}$ for $H=21 \mathrm{~mm}$. The data are well fitted to Eq. (B3). With $\Delta \rho \simeq 400 \mathrm{~kg} . \mathrm{m}^{-3}$ and $\phi \simeq 0.5$, as estimated in Sec. II A, this yields $\mu=0.13$, which seems to be a reasonable value, and is consistent with the order of magnitude found below for the case of dominant capillary pressure.

We now show in Fig. 18 the results obtained when studying a $29 \mathrm{wt} \%$ suspension prepared as explained in Sec. II A. We first note that the torque mostly evolves within a few units of strain when the material is sheared, as expected for a dila-

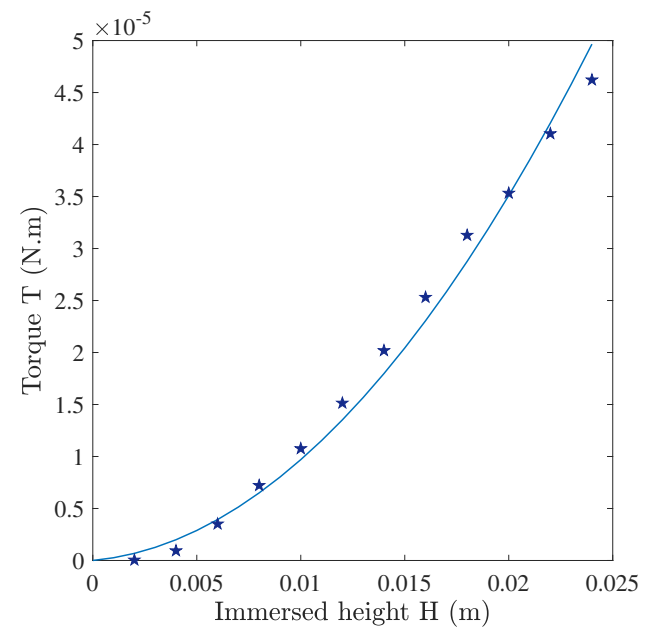

FIG. 17. Torque vs. immersed height for a settled suspension of silica particles. The line is a fit of the data to Eq. (B3), with $\mu=0.13$.

tant material (an example is shown in Fig. 19). However, in addition, an apparent long term decrease of the stress in time was observed and attributed to the progressive digging of the free surface, due to the plastic behavior of this material: we thus chose to report the torque values reached for a constant shear strain $\gamma=10$, which should provide a good estimate of the steady-state behavior. The values reported here are thus higher than those found with the shear rate ramp in Sec. III A.

Initially the suspension is homogeneous. Upon shear, shear banding is expected and clearly observed. The critical volume fraction of the granular material has been estimated as $24 \mathrm{wt} \%$; so dilation is expected both in the radial and vertical directions. This is obvious from the aspect of the free surface, from which particles are observed to protrude. This protrusion is expected to provide a maximum confining capillary pressure $p_{\text {cap }} \simeq \Gamma_{t} / r_{p} \simeq 5000 \mathrm{~Pa}$, which is much higher than the gravity stress for the $H$ investigated. Consistently, the resulting shear stresses are $\sim 100$ times higher than when gravity is dominant, and we observe in Fig. 18 an affine increase of the torque $T$ with $H$, which is well fitted to Eq. (B4) with a friction coefficient $\mu=0.12$. This is consistent with the value of $\mu$ found above for dominant gravity. Note however that a truly quantitative determinations of $\mu$ cannot be expected here given the gross approximation on $p_{\text {cap }}$ and on the effective density of our particles.

To further evidence the role of the capillary forces at the free surface, we have performed additional experiments in which we have prepared a $29 \mathrm{wt} \%$ suspension as above, immersed the vane tool at various heights $H$, and added water on top of the material to suppress capillary forces before rotating the vane tool. In Fig. 19, we plot the torque vs. angular displacement measured in these conditions for a small height $H=6 \mathrm{~mm}$, and compare the results to those observed for the sediment and for the $29 \mathrm{wt} \%$ suspension with no added water.

We observe a long transient, with two main features: (i) the torque tends to a steady-state value which is much lower than for the suspension with no added water, and which is close to that observed for the sediment, and (ii) there is an 


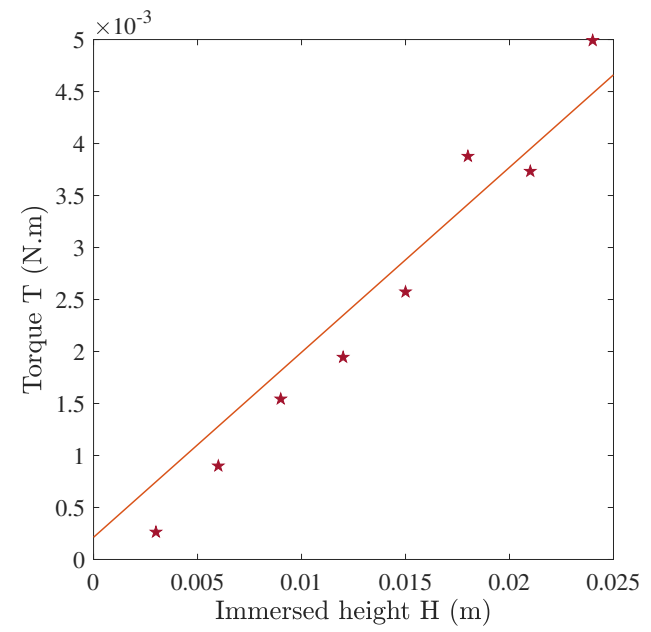

FIG. 18. Torque vs. immersed height for a $29 \mathrm{wt} \%$ suspension of silica particles. The line is a fit of the data to Eq. (B4), with $\mu=0.12$.
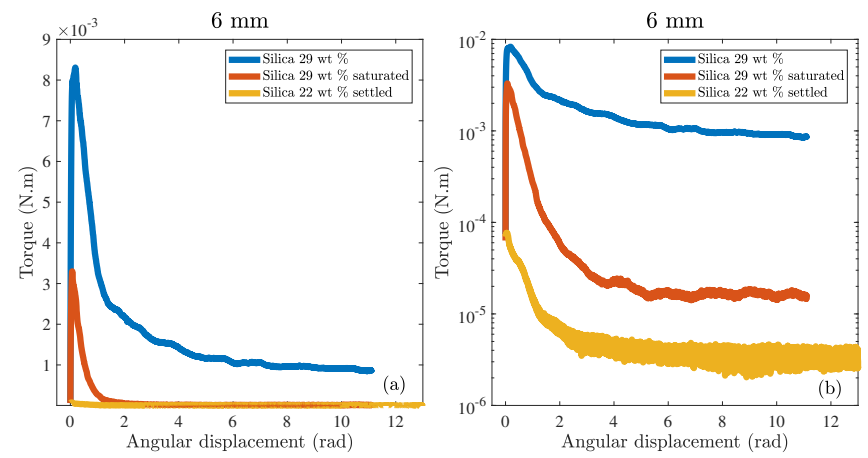

FIG. 19. Torque vs. angular displacement of the vane tool measured at a macroscopic shear rate of $0.1 \mathrm{~s}^{-1}$ for an immersed height $H=6 \mathrm{~mm}$ in three different materials (see legend): a settled $22 \mathrm{wt} \%$ suspension, a $29 \mathrm{wt} \%$ suspension, and a $29 \mathrm{wt} \%$ suspension on top of which water is added (left: linear scale, right: semi-logarithmic scale).

initial peak of same order of magnitude as for the suspension with no added water. The peak is characteristic of the initial dilation and is hard to interpret quantitatively; its large value is probably due to the large initial internal stresses due to the loading of the suspension. The long term decrease to a very low value confirms the absence of significant cohesive forces in the prepared $29 \mathrm{wt} \%$ suspension, and is due to the dilation that is now allowed in the vertical direction with a vertical stress scale set by gravity instead of capillarity.

Data are shown here at low $H$ only: at larger values, there are very long transients, which point to the persistence of internal stresses and to the difficulty to dilate in the vertical direction once these very dense suspensions have been prepared. We did not study this point in more detail.

Let us finally note that, within our experimental conditions, in the frictional regime, the bottom contribution is of order $5 \%$. For viscous flows, this bottom contribution is smaller. For the sake of simplicity, we did not take this contribution into account when computing stresses in Sec. III, as it would require a regime-dependent correction.

\section{Appendix C: Scaling of $\dot{\gamma}_{c}$ and $\sigma_{c}$ with $A$ and $f$}

In Sec. IIIC, we have shown that there is a fairly linear correlation between $\dot{\gamma}_{c}, \sigma_{c}$ and the vibration stress $\sigma_{v}=$ $1 / 2 \rho_{s} A^{2}(2 \pi f)^{2}$. In order to better show their independent scaling with $A$ and $f$, in Figs. 20a and b we plot $\sigma_{c}$ versus $A$ for a given value of $f$, and versus $f$ for a given value of $A$ ( $\dot{\gamma}_{c}$ values follow by dividing $\sigma_{c}$ by the average viscosity plateau value $10 \mathrm{~Pa} . \mathrm{s})$. All data are consistent with a $A^{2}$ and a $f^{2}$ dependence.
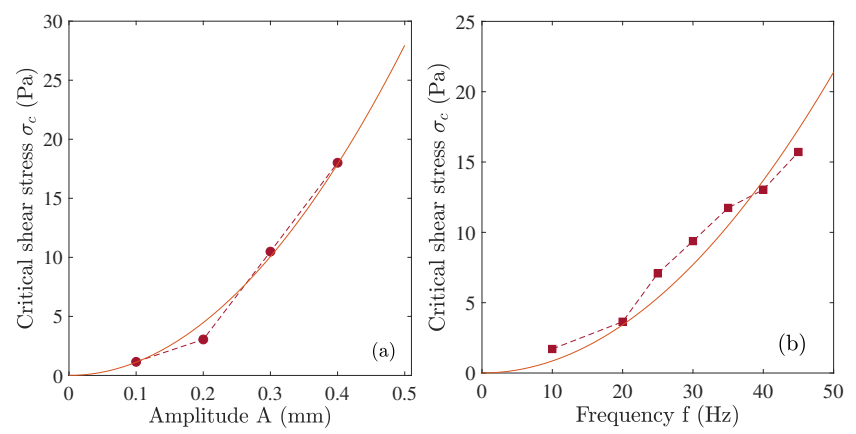

FIG. 20. Critical shear stress $\sigma_{c}$ of a $29 \mathrm{wt} \%$ silica suspension: (a) as a function of the vibration amplitude $A$ for a given value of the frequency $f=50 \mathrm{~Hz}$, and (b) as a function of the vibration frequency $f$ for a given value of the amplitude $A=500 \mu \mathrm{m}$. The solid lines are fits of the data to quadratic forms.

\section{Appendix D: Flow curves of a $28 w t \%$ and a $26 w t \%$ suspension with and without vibrations}

Fig. 21 displays the flow curves of a $28 \mathrm{wt} \%$ and a $26 \mathrm{wt} \%$ suspension for various values of the amplitude $A$ and frequency $f$ of vibrations. The $28 \mathrm{wt} \%$ suspension shows the same behavior as that observed for the $29 \mathrm{wt} \%$ suspension and discussed in detail in Sec. III C. For the $26 \mathrm{wt} \%$ suspension, sharp but continuous shear thickening is observed; the transition is shifted to higher $\dot{\gamma}_{c}$ when increasing $\sigma_{v}$, as observed for the other particle fractions.

\section{Appendix E: Shear reversal vs. vibrations for a $27 \mathrm{wt} \%$ and a $24 \mathrm{wt} \%$ suspension}

The minimum viscosity $\eta_{\min }$ achieved during the shearreversal experiments is plotted versus the applied shear stress in Fig. 22 for the $27 \mathrm{wt} \%$ and the $24 \mathrm{wt} \%$ suspension, together with the steady state viscosity; the flow curves obtained from a shear rate ramp with or without vibrations are also displayed. The same behavior is observed as for the $26 \mathrm{wt} \%$ suspension (Fig. 11), which is discussed in detail in Sec. III E. 

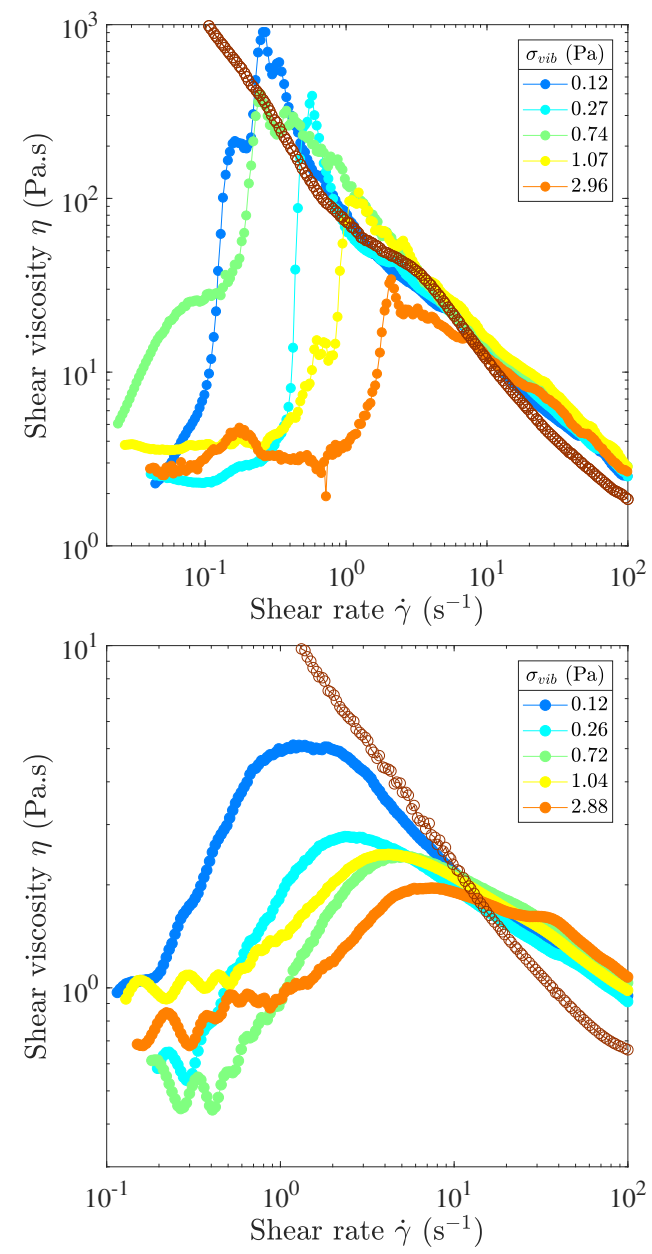

FIG. 21. Flow curves of a $28 \mathrm{wt} \%$ (top) and a $26 \mathrm{wt} \%$ (bottom) silica suspension: shear viscosity $\eta$ vs. shear rate $\dot{\gamma}$ for different values of vibration stress $\sigma_{v}$. Empty symbols correspond to the nonvibrated samples.

The main result is that the viscosity of the vibrated suspension at low shear rate is similar to the minimum viscosity in the shear-reversal experiments. This strongly suggests that vibrated suspensions are equivalent to suspensions of frictionless particles.

\section{Appendix F: Prediction of the Wyart and Cates (2014) model}

The Wyart and Cates [16] model for non-Brownian frictional spheres considers that interparticle contacts are either lubricated or frictional, and that the jamming volume fraction $\phi_{j}\left(f_{c}\right)$ of a suspension is a function of the fraction $f_{c}$ of frictional contacts. Noting that in the absence of contacts $\left(f_{c}=0\right)$, $\phi_{j}$ should be equal to the random close packing $\phi_{\text {rcp }}$ and that when all contacts are frictional $\left(f_{c}=1\right), \phi_{j}=\phi_{m}$ (where $\phi_{m}$ depends on the value of the interparticle friction coefficient $\mu_{p}$ ), a simple interpolation function was proposed:

$$
\phi_{j}\left(f_{c}\right)=f_{c} \phi_{m}+\left(1-f_{c}\right) \phi_{\mathrm{rcp}}
$$
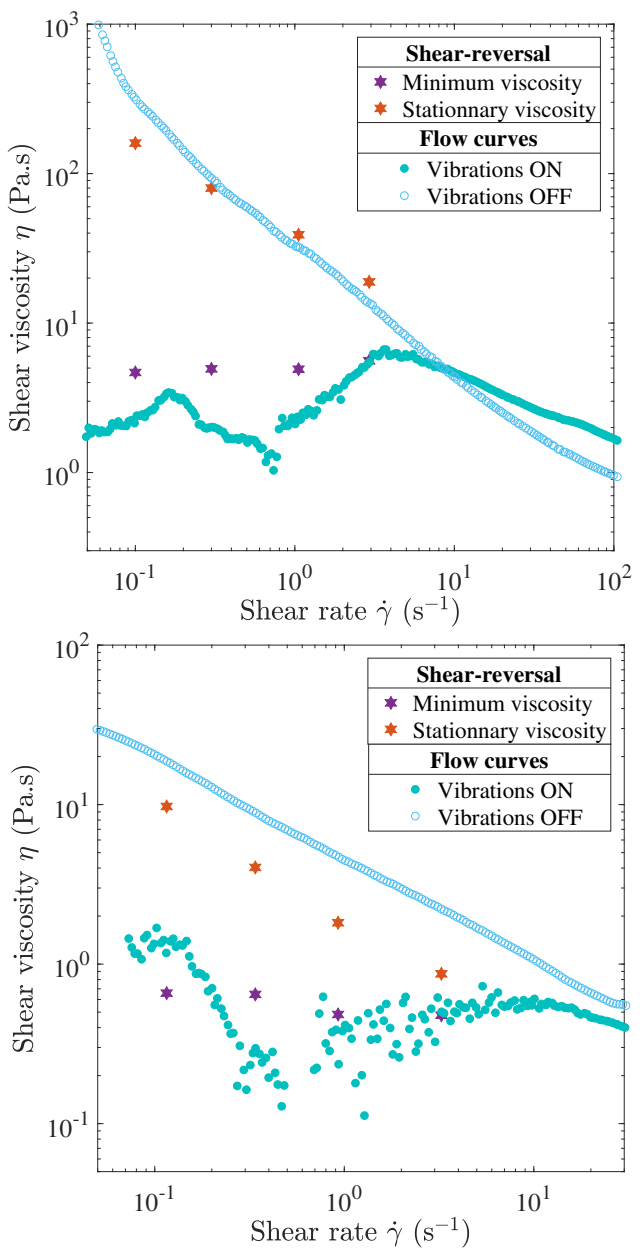

FIG. 22. Shear viscosity versus shear rate for a $27 \mathrm{wt} \%$ (top) and a $24 \mathrm{wt} \%$ (bottom) suspension. Stars: steady-state and minimum viscosity obtained from shear-reversal experiments. Circles: flow curves obtained from a shear rate ramp with or without vibrations (here $\sigma_{v}=3 \mathrm{~Pa}$ ).

For suspensions of volume fraction $\phi<\phi_{j}\left(f_{c}\right)$, Eq. (F1) is used in the macroscopic description of the viscous behavior of the material

$$
\sigma=\eta_{f} \eta_{r}\left(\phi / \phi_{j}\left(f_{c}\right)\right) \dot{\gamma}
$$

with $\eta_{f}$ the interstitial fluid viscosity. The relative viscosity $\eta_{r}$ of the suspension is given by

$$
\eta_{r}\left(\phi / \phi_{j}\left(f_{c}\right)\right)=\frac{C}{\left(1-\phi / \phi_{j}\left(f_{c}\right)\right)^{2}}
$$

where $C$ is a free parameter; $C \sim 1$ is usually reported for spheres $[3,12]$.

The fraction of frictional contacts is expressed as

$$
f_{c}=\exp \left(-\left(\sigma_{r} / \sigma\right)^{\beta}\right)
$$

where $\sigma_{r}$ is the typical repulsive stress to overcome to form contacts, and is of the order of the critical stress $\sigma_{c}$ for shearthickening. In Eq. (F4), $\beta$ is a constant parameter; $\beta=1$ was 
found in simulations by Singh et al. [12] whereas $\beta=0.85$ is reported in experiments by Guy, Hermes, and Poon [18]. Altogether, these equations are used to model the steady-state shear-thickening behavior.

To illustrate this model on our systems, as discussed in Sec. IV C, we use

$$
\sigma_{r}=\alpha \sigma_{v}
$$

We use Eqs. (F1)-(F4) with $\beta=1$, while noting that $\phi / \phi_{j}\left(f_{c}\right)=\phi^{\mathrm{wt}} / \phi_{j}^{\mathrm{wt}}\left(f_{c}\right), \phi_{j}^{\mathrm{wt}}(0)=\phi_{\mathrm{rcp}}^{\mathrm{wt}}=0.303$ and $\phi_{j}^{\mathrm{wt}}(1)=$ $\phi_{m}^{\mathrm{wt}}=0.24$. We use $C=18$ as found in Fig. 9 .

Since we have studied a suspension of volume fraction $\phi_{j}(1)<\phi<\phi_{j}(0)$, Eqs. (F2) and (F3) can be used only as long as $\phi<\phi_{j}\left(f_{c}\right)$. For $\phi_{j}\left(f_{c}\right) \leq \phi$ we can either consider the material as jammed or write the apparent behavior as $\sigma \simeq \sigma_{y}$ as explained and observed for the shear jammed material. Since we work at imposed macroscopic shear rate, we have chosen this last option with the $\sigma_{y}$ values found experimentally. Of course, this works only if the critical stress for shear jamming is lower than $\sigma_{y}$, which is the case in all of our experiments.

The model predictions are plotted and discussed in Sec. IV C. Fair agreement with the experimental data is found when $\alpha=6$ is used in Eq. (F5).

\section{REFERENCES}

[1] F. Toussaint, C. Roy, and P.-H. Jézéquel, "Reducing shear thickening of cement-based suspensions," Rheologica acta 48, 883-895 (2009).

[2] E. Blanco, D. J. Hodgson, M. Hermes, R. Besseling, G. L. Hunter, P. M. Chaikin, M. E. Cates, I. Van Damme, and W. C. Poon, "Conching chocolate is a prototypical transition from frictionally jammed solid to flowable suspension with maximal solid content," Proceedings of the National Academy of Sciences 116, 10303-10308 (2019).

[3] E. Guazzelli and O. Pouliquen, "Rheology of dense granular suspensions," Journal of Fluid Mechanics 852, P1 (2018).

[4] J. F. Morris, "Shear Thickening of Concentrated Suspensions: Recent Developments and Relation to Other Phenomena," Annual Review of Fluid Mechanics 52, 121-144 (2020).

[5] D. J. M. Hodgson, M. Hermes, E. Blanco, and W. C. K. Poon, "Granulation and suspension rheology: a unified treatment," arXiv:1907.10980 [cond-mat] (2019).

[6] Y. Forterre and O. Pouliquen, "Flows of dense granular media," Annu. Rev. Fluid Mech. 40, 1-24 (2008).

[7] F. Boyer, E. Guazzelli, and O. Pouliquen, "Unifying Suspension and Granular Rheology," Physical Review Letters 107, 188301 (2011).

[8] A. Schofield and P. Wroth, Critical state soil mechanics (McGraw-hill, 1968).

[9] B. Andreotti, Y. Forterre, and O. Pouliquen, Granular media: between fluid and solid (Cambridge University Press, 2013).

[10] S. Gallier, E. Lemaire, F. Peters, and L. Lobry, "Rheology of sheared suspensions of rough frictional particles," Journal of Fluid Mechanics 757, 514-549 (2014).

[11] W. Chèvremont, B. Chareyre, and H. Bodiguel, "Quantitative study of the rheology of frictional suspensions: Influence of friction coefficient in a large range of viscous numbers," Physical Review Fluids 4, 064302 (2019).

[12] A. Singh, R. Mari, M. M. Denn, and J. F. Morris, "A constitutive model for simple shear of dense frictional suspensions," Journal of Rheology 62, 457-468 (2018).

[13] P.-E. Peyneau and J.-N. Roux, "Frictionless bead packs have macroscopic friction, but no dilatancy," Physical Review E 78, 011307 (2008).

[14] R. Lespiat, S. Cohen-Addad, and R. Höhler, "Jamming and Flow of Random-Close-Packed Spherical Bubbles: An Analogy with Granular Materials," Physical Review Letters 106, 148302 (2011).
[15] N. Fernandez, R. Mani, D. Rinaldi, D. Kadau, M. Mosquet, H. LomboisBurger, J. Cayer-Barrioz, H. J. Herrmann, N. D. Spencer, and L. Isa, "Microscopic mechanism for shear thickening of non-brownian suspensions," Physical review letters 111, 108301 (2013).

[16] M. Wyart and M. Cates, "Discontinuous Shear Thickening without Inertia in Dense Non-Brownian Suspensions," Physical Review Letters 112, 098302 (2014).

[17] R. Mari, R. Seto, J. F. Morris, and M. M. Denn, "Shear thickening, frictionless and frictional rheologies in non-brownian suspensions," Journal of Rheology 58, 1693-1724 (2014).

[18] B. M. Guy, M. Hermes, and W. C. K. Poon, "A unified description of the rheology of hard-particle suspensions," Physical Review Letters 115, 088304 (2015).

[19] J. Comtet, G. Chatté, A. Niguès, L. Bocquet, A. Siria, and A. Colin, "Pairwise frictional profile between particles determines discontinuous shear thickening transition in non-colloidal suspensions," Nature Communications 8, 15633 (2017).

[20] G. Bossis, P. Boustingorry, Y. Grasselli, A. Meunier, R. Morini, A. Zubarev, and O. Volkova, "Discontinuous shear thickening in the presence of polymers adsorbed on the surface of calcium carbonate particles," Rheologica Acta 56, 415-430 (2017).

[21] N. Y. Lin, B. M. Guy, M. Hermes, C. Ness, J. Sun, W. C. Poon, and I. Cohen, "Hydrodynamic and contact contributions to continuous shear thickening in colloidal suspensions," Physical review letters 115, 228304 (2015).

[22] N. Y. Lin, C. Ness, M. E. Cates, J. Sun, and I. Cohen, "Tunable shear thickening in suspensions," Proceedings of the National Academy of Sciences 113, 10774-10778 (2016).

[23] C. Ness, R. Mari, and M. E. Cates, "Shaken and stirred: Random organization reduces viscosity and dissipation in granular suspensions," Science advances 4, eaar3296 (2018).

[24] R. Niu, M. Ramaswamy, C. Ness, A. Shetty, and I. Cohen, "Tunable solidification of cornstarch under impact: How to make someone walking on cornstarch sink," Science Advances 6, eaay6661 (2020).

[25] C. Hanotin, S. Kiesgen de Richter, P. Marchal, L. J. Michot, and C. Baravian, "Vibration-induced Liquefaction of Granular Suspensions," Physical Review Letters 108, 198301 (2012).

[26] C. Hanotin, S. Kiesgen de Richter, L. J. Michot, and P. Marchal, "Viscoelasticity of vibrated granular suspensions," Journal of Rheology 59, 253-273 (2015).

[27] C. Hanotin, P. Marchal, L. J. Michot, C. Baravian, and S. Kiesgen de Richter, "Dynamics of vibrated granular suspensions probed by mechanical spectroscopy and diffusing wave spectroscopy measurements," Soft Matter 9, 9352 (2013).

[28] N. Gaudel, S. Kiesgen de Richter, N. Louvet, M. Jenny, and S. SkaliLami, "Bulk and local rheology in a dense and vibrated granular suspension," Physical Review E 96, 062905 (2017).

[29] A. Singh, C. Ness, R. Seto, J. J. de Pablo, and H. M. Jaeger, "Shear thickening and jamming of dense suspensions: the "roll" of friction," Physical Review Letters 124, 248005 (2020).

[30] J. Laurens, J. Jolly, G. Ovarlez, H. Fay, T. Chaussée, and P. Sotta, “Competitive adsorption between a polymer and solvents onto silica," Langmuir 36, 7669-7680 (2020).

[31] A. Fall, F. Bertrand, D. Hautemayou, C. Mezière, P. Moucheront, A. Lemaître, and G. Ovarlez, "Macroscopic Discontinuous Shear Thickening versus Local Shear Jamming in Cornstarch," Physical Review Letters 114, 098301 (2015).

[32] N. Estrada, E. Azéma, F. Radjai, and A. Taboada, "Identification of rolling resistance as a shape parameter in sheared granular media," Physical Review E 84, 011306 (2011).

[33] N. Estrada, A. Taboada, and F. Radjai, "Shear strength and force transmission in granular media with rolling resistance," Physical Review E 78, 021301 (2008).

[34] P. Marchal, N. Smirani, and L. Choplin, "Rheology of dense-phase vibrated powders and molecular analogies," Journal of Rheology 53, 1-29 (2009).

[35] K. J. Gutam, V. Mehandia, and P. R. Nott, "Rheometry of granular materials in cylindrical couette cells: Anomalous stress caused by gravity and shear," Physics of Fluids 25, 070602 (2013). 
[36] A. Aitt-Kadi, P. Marchal, L. Choplin, A.-S. Chrissemant, and M. Bousmina, "Quantitative Analysis of Mixer-Type Rheometers using the Couette Analogy," The Canadian Journal of Chemical Engineering 80, 11661174 (2002).

[37] F. Da Cruz, F. Chevoir, D. Bonn, and P. Coussot, "Viscosity bifurcation in granular materials, foams, and emulsions," Physical Review E 66, 051305 (2002).

[38] F. Peters, G. Ghigliotti, S. Gallier, F. Blanc, E. Lemaire, and L. Lobry, "Rheology of non-brownian suspensions of rough frictional particles under shear reversal: A numerical study," Journal of rheology $\mathbf{6 0}, 715-732$ (2016).

[39] B. Saint-Michel, S. Manneville, S. Meeker, G. Ovarlez, and H. Bodiguel, "X-ray radiography of viscous resuspension," Physics of Fluids 31, 103301 (2019).

[40] L. A. A. Yahia, T. M. Piepke, R. Barrett, A. Ozel, and R. Ocone, "Development of a virtual couette rheometer for aerated granular material," AIChE Journal 66, e16945 (2020).

[41] C. Ancey and P. Coussot, "Transition from frictional to viscous regime for granular suspensions," Comptes Rendus de l'Academie des Sciences Series IIB Mechanics Physics Astronomy 5, 515-522 (1999).
[42] M. Cates, M. Haw, and C. Holmes, "Dilatancy, jamming, and the physics of granulation," Journal of Physics: Condensed Matter 17, S2517 (2005).

[43] P. Schall and M. van Hecke, "Shear bands in matter with granularity," Annual Review of Fluid Mechanics 42 (2010).

[44] J. Vermant, P. Moldenaers, J. Mewis, M. Ellis, and R. Garritano, "Orthogonal superposition measurements using a rheometer equipped with a force rebalanced transducer," Review of scientific instruments 68, 40904096 (1997).

[45] G. Ovarlez, Q. Barral, and P. Coussot, "Three-dimensional jamming and flows of soft glassy materials," Nature materials 9, 115-119 (2010).

[46] G. Ovarlez, F. Bertrand, P. Coussot, and X. Chateau, "Shear-induced sedimentation in yield stress fluids," Journal of Non-Newtonian Fluid Mechanics 177, 19-28 (2012).

[47] R. Mari, R. Seto, J. F. Morris, and M. M. Denn, "Discontinuous shear thickening in brownian suspensions by dynamic simulation," Proceedings of the National Academy of Sciences 112, 15326-15330 (2015).

[48] G. Chatté, J. Comtet, A. Niguès, L. Bocquet, A. Siria, G. Ducouret, F. Lequeux, N. Lenoir, G. Ovarlez, and A. Colin, "Shear thinning in non-brownian suspensions," Soft matter 14, 879-893 (2018).

[49] L. Lobry, E. Lemaire, F. Blanc, S. Gallier, and F. Peters, "Shear thinning in non-brownian suspensions explained by variable friction between particles," Journal of Fluid Mechanics 860, 682-710 (2019). 